\title{
A Philippine Tertiary Hospital Cross-sectional and Registry Feasibility Study: Gout Clinical Case Scenario
}

\author{
${ }^{1}$ Tricity Medical Center, Pasig City \\ ${ }^{2}$ Philippine General Hospital, University of the Philippines Manila \\ ${ }^{3}$ Makati Medical Center, Makati City \\ ${ }^{4}$ Asian Hospital and Medical Center, Muntinlupa City
}

Ronaldo Q. De Vera, MD, ${ }^{1}$ Grace G. Penserga, MD² and Jose Paulo P. Lorenzo, MD ${ }^{2,3,4}$

\begin{abstract}
Background. Gout is one of the most common arthritides affecting Filipinos; yet, there is a lack of updated local data and Clinical Practice Guidelines.

Objective. To describe Clinical Case Scenario (CCS) of Filipino patients with gout in a tertiary referral hospital seen over a year.

Design. Cross-sectional study.

Methods. Patients' characteristics, risk factors, disease course, management, and CCS were obtained by a rheumatologist using a questionnaire. Descriptive statistics were used.

Results. One hundred eight patients were included with a median age of 58 (range 26-80) years. 106 were male (98\%); and, 2 were female (2\%) who were menopause and had chronic kidney disease (CKD). Most prevalent CCS were stages 9 (29\%), 1 (16\%), and 2 (15\%). The majority of cases had tophi and belonged to CCS 4-9 (62\%). This signifies that most patients had advanced gout. Consistent with international and local data: almost half had hypertension (46\%), a third had CKD (36\%). Most were ethanol drinkers (65\%) and smokers (57\%). Unexpectedly, not many were obese (10\%) or had metabolic syndrome (2\%). The initial joint involved was the ankle (52\%) rather than the first metatarsophalangeal joint (40\%). Almost half of the patients presented with two or more joint involvement (46\%) than monoarthritis (54\%). Patients with acute flare were most commonly prescribed NSAIDs (77\%), followed by colchicine (62\%). Most were prescribed allopurinol (44\%) compared with febuxostat (37\%) for urate-lowering therapy. Only $16 \%$ received patient education. Medication compliance was $65 \%$, but followup compliance was less than $18 \%$. Comparing the Filipino clinical profile to historical data suggests an increased incidence of gout in the young and an increase in comorbidity prevalence.
\end{abstract}

Conclusion. This study reports a cohort of Filipino gout patients. Comorbidities are similar to world figures but differ in the low incidence of obesity and metabolic syndrome. It also differs from literature in having the ankle as the most common initial joint presentation. Management and compliance were also described. As a pilot study for a registry, this study can be implemented at different institutions to broaden and monitor the ever-changing Filipino gout profile.

Recommendation. A larger sample size and a more extended observation period are recommended to estimate gout CCS prevalence, flare risk factors, and treatment response more accurately. Other outcomes that can be measured are mortality rates and etiologies for each CCS.

Key Words: Filipino, gout, classification, comorbidity, registry

Corresponding author: Ronaldo Q. De Vera, MD

Tricity Medical Center

Raymundo Avenue, Pasig City

Metro Manila 1607, Philippines

Email: aldodevera.md@gmail.com 


\section{INTRODUCTION}

Gouty arthritis is one of the most common arthritides affecting man today. Gout is a spectrum of clinical and pathologic features built on a foundation of excess uric acid where there is tissue deposition of monosodium urate (MSU) crystals. ${ }^{1}$ It is characterized by recurrent attacks of acute inflammatory arthritis due to MSU deposition in joints. According to the 2015 American College of RheumatologyEuropean League Against Rheumatism (ACR-EULAR) Gout Classification Criteria, gout is considered when the patient presents one episode of peripheral joint or bursa swelling pain, or tenderness. ${ }^{2}$ The typical pattern of joint involvement (metatarsophalangeal (MTP) joint involvement), joint characteristics (erythema, exquisite tenderness, loss of function secondary to pain), the time course of episodes (complete resolution between symptomatic episodes), and clinical evidence of tophi on physical examination are criteria supportive of gout. Laboratory values would show elevated serum uric acid and synovial fluid MSU crystals. The finding of a double contour sign in an ultrasound study, urate deposition in computed tomography, and rat-bite erosions in the $\mathrm{x}$-ray contribute to gout diagnosis.

In the Philippines, gout is the most common inflammatory arthritis, with an overall prevalence of $1.6 \% .{ }^{3,4}$ It is primarily a disease of males but may also affect females, especially in the postmenopausal age group when uricosuric protection from estrogen wanes., ${ }^{3,5-8}$ Males who experience high serum uric acid levels for more extended periods increase their predisposition to uric acid deposition and, ultimately, gout disease. Gout is 2.6-fold higher in males than females, and its incidence increases with age, with a plateau after 70 years old. ${ }^{9}$

Gout usually develops in middle-aged men, but its age of onset has been seen to decrease with some juvenile gout associated with overweight and heredity background. ${ }^{10,11}$ Certain behaviors such as alcohol beverage drinking and diet preference may aggravate gout disease and trigger attacks. ${ }^{12}$ Patients are also at a higher risk for gout when they have co-morbidities such as chronic kidney disease, cardiovascular disease, diabetes, and metabolic syndrome. ${ }^{13}$ Medication such as diuretics, low dose aspirin, and antituberculosis treatment are known to increase uric acid and trigger a gout attack.

To direct management, a patient with gout is traditionally classified according to four stages: asymptomatic hyperuricemia, acute gout, intercritical/interval gout, and chronic tophaceous gout. This is also how the 2008 Philippine Clinical Practice Guideline (CPG) defines gout's clinical stages. ${ }^{14}$ More recently, the American College of Rheumatology ("ACR") Core Expert Panel created the nine fundamental Clinical Case Scenario/Stage(CCS) during the formation of the 2012 ACR Guidelines for Management of Gout. ${ }^{15}$ The CCS reflects the broad differences in the severity of gout and its clinical manifestations. There are three distinct groups of CCS: Group A - no tophi on physical examination but with intermittent symptoms of gout (CCS 1 - 3); Group B - at least one tophus on physical examination with intermittent symptoms of acute gouty arthritis (CCS 4 - 6); and, Group C - at least one tophus on physical examination with chronic joint symptoms due to synovitis attributable to gout, or patients with unstable tophi (CCS 7 -9). Each clinical scenario exhibits differences in the frequency of symptoms in a year and the presence or extent of chronic findings such as tophi or synovitis. The nine different CCS can be viewed as a spectrum of gout, with CCS 1 being the mildest and CCS 9 the most severe.

Regardless of stage, untreated gout may progress to joint destruction, uric acid deposition in other tissues referred to as tophi, genitourinary stone formation, and kidney failure. ${ }^{16,17}$ Classifying a patient with gout according to CCS should allow for a more individualized treatment regimen. Patient education on diet, weight loss, lifestyle changes, assessment of co-morbidities and their treatment, and analysis of medications with discontinuation of unnecessary prescriptions are essential targets in gout management. ${ }^{15,18}$

Despite gout being the most common cause of inflammatory arthritis in the Philippines, increased incidence in the young, and studies showing gout or hyperuricemia as a substantial risk comorbid, the last local CPG was published more than ten years ago. ${ }^{3,10,14}$ To properly approach gout in the Philippine setting, physicians need local and updated gout data on patients' clinical characteristics, response to medical management, and treatment outcomes. A clinical data registry records information about patients' health status and the health care they receive over some time. ${ }^{19}$ The creation of a dedicated gout registry will provide the foundation for modernizing the Philippine CPG and its upkeep. In the long term, it will improve the quality of care of patients and prevent complications directly from gout and indirectly from gout's associated co-morbidities.

This study aimed to describe a Filipino gout patient by the different phases of gout classified by the Philippine CPG and the chronicity of pain and number of flares experienced in the past year CCS as defined by the ACR. This study set the stage for implementing an integrated gout registry among hospitals in the Philippines.

\section{METHODOLOGY}

\section{Study Design, Setting, and Population}

This was a cross-sectional, descriptive study of 108 patients seen in the Philippine General Hospital, Section of Rheumatology service, with data collected for one year. The research protocol was submitted to the University of the Philippines Manila Research and Ethics Board for approval before its implementation. The difficulties, nuances experienced, and expenses incurred were considered for improving and checking the feasibility of an integrated gout registry in the Philippines. 
All consenting Filipino patients fulfilling the 2015 ACR-EULAR Gout Classification Criteria were included (Appendix A). Patients initially diagnosed with gout but eventually diagnosed to have another form of arthritis causing similar symptoms were excluded.

\section{Data Collection}

The co-investigator, a rheumatology fellow-in-training, performed history taking and a musculoskeletal exam at the time of consult and assessed the current disease state. A questionnaire was used to collect four different data sets: first, the patient's baseline characteristics, including comorbidities, the age of gout diagnosis, initial manifestations, previous check-ups, medications, and procedures. Second, the CCS was determined through history and physical examination (Appendix B). Third, disease state was assessed, which involved identifying a flare, triggers of the flare, previous management and response, and common laboratory findings requested in gout. The choice of treatment given by the rheumatologist was also recorded in this part. Lastly, the disease state on follow-up was determined to assess any worsening or improvement of the previous status.

\section{Statistical Analysis}

Descriptive statistics was used to summarize the general and clinical characteristics of the participants. Frequency and proportion were used for nominal variables, median and range for ordinal variables, and mean and standard deviation for interval/ratio variables. All valid data were included in the analysis. Missing variables were neither replaced nor estimated. STATA 15.0 was used for data analysis.

\section{RESULTS}

A total of 108 patients were analyzed, with median age of 58 (range 26-80) years (Table 1). Only two were female (2\%); both had chronic kidney disease, menopause, and started experiencing gout symptoms at 55 years old. The most prevalent comorbidities in the population were hypertension (43\%) and chronic kidney disease (33\%). Four patients had a previous stroke (4\%), and 15 patients have a history of coronary heart disease (14\%). A family history of gout was present in 20 patients (19\%). More than half were alcohol beverage drinkers $(60 \%)$ or smokers $(53 \%)$.

Table 2 shows the age of first symptoms and CCS distribution of the 108 patients. Most patients experienced their first gout symptoms in $40-44$ years old (16.6\%). It is noted that below age 30, there were already 18 patients who manifested gouty attacks. Eleven of these patients belong to the highest CCS group.

Symptoms of gout were first noticed at age $42 \pm 13$ years (Table 1). The average age at diagnosis of gouty arthritis was $49 \pm 12$ years. While approximately a third of patients consulted on the same year their gout symptoms appeared, four patients took $30-40$ years to consult. Consultation with a healthcare worker did not immediately translate to having a gout diagnosis, and three patients took $10-14$ years after the first consultation before being diagnosed with gout.

Forty-two patients (38.89\%) consulted immediately on presentation of first symptoms of gout, with 29 patients diagnosed within the same year and 13 patients diagnosed only after $1-14$ years (with an average of 5.15 , mode of 1 , and median of 3 years). Eighty patients (74.1\%) were diagnosed correctly with gout within the same year of their

Table 1. Demographic and Clinical Characteristics ( $n=108)$

\begin{tabular}{|c|c|}
\hline & Median (Range); Mean \pm SD \\
\hline Age, years & 58 (26-80); $55.31 \pm 11.93$ \\
\hline Age at symptom onset, years & 41 (15-72); $41.91 \pm 12.94$ \\
\hline Age at first check-up, years & $50(23-72) ; 47.68 \pm 11.82$ \\
\hline Age at diagnosis, years & 50 (23-73); $48.87 \pm 11.88$ \\
\hline $\mathrm{BMI}\left(\mathrm{kg} / \mathrm{m}^{2}\right)$ & 23.95 (15.6-36.5); $24.09 \pm 4.27$ \\
\hline Weight (kg) & 68.5 (39-110); $67.46 \pm 12.17$ \\
\hline \multirow[t]{2}{*}{ Height $(\mathrm{cm})$} & 167.6 (124.9-195); $167.52 \pm 8.59$ \\
\hline & Frequency (\%) \\
\hline \multicolumn{2}{|l|}{ Sex } \\
\hline Male & $106(98.15)$ \\
\hline Female & $2(1.85)$ \\
\hline \multicolumn{2}{|l|}{ Comorbidities } \\
\hline Hypertension & $46(42.59)$ \\
\hline Chronic kidney disease & $36(33.33)$ \\
\hline Diabetes mellitus & $17(15.74)$ \\
\hline Coronary heart disease & $15(13.89)$ \\
\hline Dyslipidemia & $12(11.11)$ \\
\hline Nephrolithiasis & $6(5.56)$ \\
\hline Stroke & $4(3.70)$ \\
\hline Metabolic syndrome & $2(1.85)$ \\
\hline Menopause & $2(1.85)$ \\
\hline Blood dyscrasia & $1(0.93)$ \\
\hline Cancer & $1(0.93)$ \\
\hline Hypothyroidism & $1(0.93)$ \\
\hline Tuberculosis & $1(0.93)$ \\
\hline \multicolumn{2}{|l|}{ Medication } \\
\hline Anti-hypertensive & $34(31.48)$ \\
\hline Lipid lowering drug & $15(13.89)$ \\
\hline Anti-diabetic & $7(6.48)$ \\
\hline Diuretic & $5(4.63)$ \\
\hline Anti-tuberculosis & $1(0.93)$ \\
\hline \multicolumn{2}{|l|}{ Family history } \\
\hline Hypertension & $36(33.33)$ \\
\hline Diabetes mellitus & $32(29.63)$ \\
\hline Gout & $20(18.52)$ \\
\hline Coronary heart disease & $20(18.52)$ \\
\hline Dyslipidemia & $5(4.63)$ \\
\hline Cancer & $4(3.70)$ \\
\hline Chronic kidney disease & $3(2.78)$ \\
\hline Blood dyscrasia & $1(0.93)$ \\
\hline \multicolumn{2}{|l|}{ Personal and social history } \\
\hline Smoker & $57(52.78)$ \\
\hline Alcohol beverage drinker & $65(60.19)$ \\
\hline
\end{tabular}


Table 2. Age at First Symptoms and Clinical Case Scenario Distribution

\begin{tabular}{crcccccccccccc}
\hline Age at first symptoms & Total & CCS1 & CCS2 & CCS3 & CCS4 & CCS5 & CCS6 & CCS8 & CCS9 & A(1-3) & B (4-6) & C(7-9) \\
\hline $15-19$ & 3 & 0 & 1 & 0 & 0 & 0 & 1 & 0 & 1 & 1 & 1 & 1 \\
$20-24$ & 6 & 0 & 2 & 0 & 0 & 0 & 0 & 0 & 4 & 2 & 0 & 4 \\
$25-29$ & 9 & 0 & 1 & 1 & 1 & 0 & 0 & 0 & 6 & 2 & 1 & 6 \\
$30-34$ & 15 & 2 & 2 & 1 & 1 & 2 & 2 & 1 & 4 & 5 & 5 & 5 \\
$35-39$ & 9 & 2 & 0 & 0 & 0 & 2 & 3 & 0 & 2 & 2 & 5 & 2 \\
$40-44$ & 18 & 2 & 2 & 1 & 2 & 5 & 0 & 1 & 5 & 5 & 7 & 6 \\
$45-49$ & 15 & 3 & 1 & 2 & 3 & 1 & 0 & 0 & 5 & 6 & 4 & 5 \\
$50-54$ & 13 & 1 & 3 & 2 & 3 & 0 & 2 & 1 & 1 & 6 & 5 \\
$55-59$ & 8 & 3 & 2 & 0 & 0 & 0 & 0 & 0 & 3 & 5 & 0 & 3 \\
$60-64$ & 7 & 3 & 1 & 1 & 0 & 2 & 0 & 0 & 0 & 5 & 2 & 0 \\
$65-69$ & 2 & 1 & 1 & 0 & 0 & 0 & 0 & 0 & 0 & 2 & 0 & 0 \\
$70-74$ & 3 & 0 & 0 & 0 & 1 & 1 & 1 & 0 & 0 & 0 & 3 \\
$>75$ & 0 & 0 & 0 & 0 & 0 & 0 & 0 & 0 & 0 & 0 & 0 \\
\hline
\end{tabular}

Table 3. Periods by Clinical Scenario (1-9)/Disease Groups (A, B, C)

\begin{tabular}{|c|c|c|c|c|c|c|c|c|c|}
\hline & \multicolumn{3}{|c|}{$\mathrm{A}(\mathrm{N}=41)$} & \multicolumn{3}{|c|}{$\mathrm{B}(\mathrm{N}=33)$} & \multicolumn{3}{|c|}{$C(\mathrm{~N}=34)$} \\
\hline & $\underset{(n=17)}{C S 1}$ & $\underset{(n=16)}{C S 2}$ & $\begin{array}{c}\text { CS3 } \\
(n=8)\end{array}$ & $\underset{(n=11)}{C S 4}$ & $\begin{array}{c}\text { CS5 } \\
(n=13)\end{array}$ & $\begin{array}{c}\text { CS6 } \\
(n=9)\end{array}$ & $\begin{array}{c}\text { CS7 } \\
(n=0)\end{array}$ & $\begin{array}{c}\text { CS8 } \\
(n=3)\end{array}$ & $\begin{array}{c}\text { CS9 } \\
(n=31)\end{array}$ \\
\hline $\begin{array}{c}\text { Diagnosed on First Consult } \\
\text { Total }=80\end{array}$ & $14(82.35)$ & $14(87.5)$ & $\begin{array}{c}5(62.5) \\
33(80.49)\end{array}$ & $8(72.73)$ & $9(69.29)$ & $\begin{array}{r}8(88.89) \\
25(75.76)\end{array}$ & 0 & $2(66.67)$ & $\begin{array}{l}20(64.52) \\
22(64.71)\end{array}$ \\
\hline $\begin{array}{l}\text { Consulted on First Symptoms } \\
\text { Total }=42\end{array}$ & $12(70.59)$ & $7(43.75)$ & $\begin{array}{c}3(37.5) \\
22(53.66)\end{array}$ & $4(36.36)$ & $4(30.377)$ & $\begin{array}{r}3(33.33) \\
11(33.33)\end{array}$ & 0 & $1(33.33)$ & $\begin{array}{l}8(25.81) \\
9(26.47)\end{array}$ \\
\hline $\begin{array}{c}\text { Diagnosed on First Symptoms } \\
\text { Total }=29\end{array}$ & 9 (52.94) & $6(37.5)$ & $\begin{array}{c}1(12.5) \\
16(39.02)\end{array}$ & 2 (18.18) & $3(23.08)$ & $\begin{array}{l}2(22.22) \\
7(21.21)\end{array}$ & 0 & 0 & $\begin{array}{l}6(19.35) \\
6(17.65)\end{array}$ \\
\hline
\end{tabular}

Table 4. Disease Duration to Diagnosis among CCS (1-9) / Disease Groups (A-C)

\begin{tabular}{|c|c|c|c|c|c|c|c|c|c|c|c|}
\hline \multirow{2}{*}{$\begin{array}{c}\text { Disease } \\
\text { Duration to } \\
\text { Diagnosis }\end{array}$} & $\begin{array}{c}\text { CS1 } \\
(n=17)\end{array}$ & $\begin{array}{c}\text { CS2 } \\
(n=16)\end{array}$ & $\begin{array}{c}\text { CS3 } \\
(n=8)\end{array}$ & $\begin{array}{c}\text { CS4 } \\
(n=11)\end{array}$ & $\begin{array}{c}\text { CS5 } \\
(n=13)\end{array}$ & $\begin{array}{c}\text { CS6 } \\
(n=9)\end{array}$ & $\begin{array}{c}\text { CS8 } \\
(n=3)\end{array}$ & $\begin{array}{c}\text { CS9 } \\
(n=31)\end{array}$ & $\begin{array}{c}A(C C S 1-3) \\
(n=41)\end{array}$ & $\begin{array}{c}B(\operatorname{CCS} 4-6) \\
(n=33)\end{array}$ & $\begin{array}{c}C(\operatorname{CCS} 7-9) \\
(n=34)\end{array}$ \\
\hline & \multicolumn{11}{|c|}{ Frequency } \\
\hline Early ( $\leq 2$ years) & $12(70.59)$ & $8(50)$ & $3(37.5)$ & $6(54.55)$ & $5(38.46)$ & $3(33.33)$ & $1(33.33)$ & $10(32.26)$ & $23(56.1)$ & $14(42.42)$ & $11(32.35)$ \\
\hline $\begin{array}{c}\text { Established } \\
\text { (>2 years) }\end{array}$ & $5(29.41)$ & $8(50)$ & $5(62.5)$ & $5(45.45)$ & $8(61.54)$ & $6(66.67)$ & $2(66.67)$ & $21(67.74)$ & $18(43.9)$ & $19(57.58)$ & $23(67.65)$ \\
\hline
\end{tabular}

first consultation; and, the remaining 28 patients $(25.9 \%)$ were diagnosed after 1 to 14 years (most frequently three years) after their first consult.

Patients who were diagnosed within the same year of their first consult, patients who consulted immediately with their first symptoms, and patients who were diagnosed within the same year of their first symptoms were clustered via CCS $(1-9)$ and their groups $(\mathrm{A}=\mathrm{CCS} 1-3, \mathrm{~B}=\mathrm{CCS} 4-6, \mathrm{CCS}$ $=7-9)($ Table 3$)$. Although trends were showing delayed diagnosis and consultations in the higher CCS, they were not significant.

Early diagnosis of gout will lead to earlier treatment and prevention of MSU deposition within joints. Early gout and established gout are defined as gout with two years or less of symptoms and gout with more than two years of symptoms, respectively. ${ }^{20}$ When analyzing the different CCS by the disease duration to diagnosis, a trend can be seen with the lower CCS having more early-than-established gout cases (Table 4). When grouped by tophi and chronicity of symptoms (A - no tophi, B - intermittent gout with tophi, C - chronic gout with tophi/unstable tophi), this trend is more evident, but there is no significant difference.

On inclusion in the study, most patients were diagnosed by medical practitioners, with Rheumatologists diagnosing 38 patients (35\%), followed by Internal Medicine with 27 patients (25\%) and General Practitioners with 20 patients (19\%) (Table 5). Four patients were diagnosed by nonmedical practitioners (4\%) before the consultation. Eighteen patients previously underwent arthrocentesis only (16.67\%), two patients had arthrocentesis and intra-articular steroid injection $(1.85 \%)$, and two patients were previously treated with intra-articular steroid injection only (1.85\%). One patient underwent surgical debridement (0.93\%).

Before inclusion, patients were taking medications that consisted mainly of nonsteroidal anti-inflammatory drugs (NSAIDs) (79\%), colchicine (62\%), allopurinol (44\%), febuxostat (37\%), and steroids (31\%) (Table 5). These may have been taken at different points during their illness and 
not necessarily at the same time. Five patients have been on multiple NSAIDs (4.6\%), and 12 patients have previously been on febuxostat and allopurinol (11.11\%). It was not stated whether patients took febuxostat and allopurinol taken simultaneously or which urate-lowering agent was started and then interchanged. Ten patients were previously medicated with herbal/alternative medicine (9\%). Medication was prescribed by Rheumatologists most commonly (53\%), followed by Internal Medicine (20\%) and General Practitioners (11\%). Twenty-one patients took medication not prescribed by medical practitioners (19\%).

Table 5. Gout History $(n=108)$

Frequency (\%)

\section{Diagnosis by}

Rheumatologist

Internal medicine

38 (35.19)

General practitioner

27 (25)

20 (18.52)

Surgeon/orthopedics

8 (7.41)

Family medicine

$7(6.48)$

Non-medical practitioner

$4(3.70)$

Other allied health practitioner

2 (1.85)

Unrecalled

$2(1.85)$

\section{Previous procedures}

Arthrocentesis

Intra-articular (IA) steroid injection

18 (16.67)

Arthrocentesis + IA steroid injection

2 (1.85)

2 (1.85)

Debridement, debulking, or excision

\section{Medications previously taken}

NSAID

1 (4.17)

Unrecalled

85 (78.70)

Diclofenac

Celecoxib

$51(47.22)$

18 (16.67)

Mefenamic

$4(3.7)$

4 (3.7)

Etoricoxib

Diclofenac + Celecoxib

Diclofenac + Etoricoxib

Celecoxib + Etoricoxib

1 (0.93)

$1(0.93)$

1 (0.93)

2 (1.85)

Celecoxib + Diclofenac + Mefenamic

75 (69.44)

35 (32.41)

28 (25.93)

12 (11.11)

67 (62.04)

34 (31.48)

$10(9.26)$

$10(9.26)$

8 (7.41)

$1(0.93)$

\section{Unrecalled}

Prescriptionist

Rheumatologist

Internal medicine

General practitioner

Family medicine

Surgeon/orthopedic

Unrecalled

Non-medical practitioner
Eighty-eight patients reported $\geq 1$ gout flare triggers (81\%), with 57 patients identifying only one trigger (53\%) and 31 patients with multiple identified triggers (29\%) (Table 6). Identified flare triggers were most commonly food (63\%), alcohol (29\%), and hospitalization (9\%), but no source was recognized in 19\%. Four patients experienced a flare due to urate-lowering therapy (ULT) (3.7\%) - allopurinol (2 patients, $1.85 \%$ ) and febuxostat (2 patients, $1.85 \%$ ).

At the outset, the joints most frequently involved overall were the ankle (52\%), first MTP joint (40\%), and the knee (30\%) (Table 7). There were no initial joint pains experienced over the spine; and, lower extremity joints were more often affected than the upper extremities.

Inflammatory joint pain on presentation was monoarticular in 53.7\%, oligoarticular in $41.7 \%$, and polyarticular in $4.63 \%$ (Table 8). In both monoarticular and oligoarticular presentation, the ankle is the most commonly involved joint, followed by the MTP. All five patients who

Table 6. Identified Gout Flare Triggers $(n=108)$

\begin{tabular}{lc} 
Number of Identified Triggers & Frequency (\%) \\
0 & \\
1 & $20(18.52)$ \\
2 & $57(52.78)$ \\
3 & $26(24.07)$ \\
4 & $3(2.78)$ \\
\hline Flare Triggers & $2(1.85)$ \\
Food & \\
Alcohol beverages & $68(62.96)$ \\
Hospitalization & $31(28.70)$ \\
Infection & $10(9.26)$ \\
Blood loss/transfusion & $4(3.70)$ \\
Allopurinol & $3(2.80)$ \\
Febuxostat & $2(1.85)$ \\
Dialysis & $2(1.85)$ \\
Anti-tuberculosis drugs & $2(1.85)$ \\
Diarrhea & $1(0.93)$ \\
Psoriasis & $1(0.93)$ \\
Surgical procedure & $1(0.93)$ \\
Unknown & $1(0.93)$ \\
\hline
\end{tabular}

Table 7. Overall Initial Joint Involvement

\begin{tabular}{lc} 
Joint Location & Frequency (\%) \\
Ankle & $56(51.85)$ \\
First metatarsophalangeal & $43(39.81)$ \\
Knee & $32(29.63)$ \\
Midfoot & $17(15.74)$ \\
Toe & $15(13.89)$ \\
Elbow & $8(7.41)$ \\
Wrist & $6(5.56)$ \\
Metacarpophalangeal & $4(3.74)$ \\
Finger & $2(1.85)$ \\
Shoulder & $1(0.93)$ \\
Spine & 0 \\
\hline
\end{tabular}


Table 8. Joint Involvement on Presentation

\begin{tabular}{lc}
\hline Monoarthritis (1 joint) $[\mathbf{n = 5 8 , 5 3 . 7 \% ]}$ & Frequency (\%) \\
Ankle & $22(37.93)$ \\
First metatarsophalangeal & $14(24.13)$ \\
Knee & $11(18.97)$ \\
Midfoot & $5(8.62)$ \\
Others* & $6(3.45)$ \\
\hline Oligoarthritis (2-3 joints) $[\mathbf{n = 4 5 , 4 1 . 6 7 \% ]}$ & \\
Ankle & $29(64.44)$ \\
First metatarsophalangeal & $24(53.33)$ \\
Knee & $19(42.22)$ \\
Toe & $11(24.44)$ \\
Midfoot & $10(22.22)$ \\
Others** & $10(22.22)$ \\
\hline Polyarthritis (4 or more) $[n=5,4.63 \%]$ & \\
First metatarsophalangeal & $5(100)$ \\
Ankle & $5(100)$ \\
Elbow & $4(80)$ \\
Toe & $3(60)$ \\
Knee & $2(40)$ \\
Midfoot & $2(40)$ \\
Wrist & $1(20)$ \\
Metacarpophalangeal & $1(20)$ \\
\hline
\end{tabular}

*Finger, toe, elbow, wrist, metacarpophalangeal

${ }^{* *}$ Wrist, elbow, metacarpophalangeal, shoulder initially presented with polyarticular joint pain involved the ankle (100\%) and first MTP joint (100\%).

The three most prevalent clinical scenarios were stages 9 (29\%), 1 (16\%), and 2 (15\%) (Table 9). There were no patients in CCS 7. Most patients in CCS 9 had unstable tophi, with 7 of 31 patients in the group having stable tophi in $\geq 5$ joints. Taken by group, Group A with no tophi and with intermittent symptoms was the most prominent population (38\%). Group C patients with chronic symptoms and stable tophi or unstable tophi followed (31\%) and Group B with tophi and intermittent symptoms (31\%). The majority of cases had tophi and belonged to CCS 4-9 (62\%). This signifies that most patients had advanced gout.

On physical exam, 67 patients (62\%) had a tophus or tophi (Table 10). Slightly more than half of the patients with tophi $(50.75 \%)$ experienced chronic symptoms rather than intermittent flares. More than a third of patients with tophi (36\%) had unstable features and belonged to CCS 9.

Table 11 presents the prevalence of tophi in the entire series of 108 patients. This considers the disease's duration from the age of first symptoms to the patient's current age during inclusion into the study. In 29 patients with a disease duration of 5 years or less, 12 patients (41\%) had tophi. More than half had tophi in patients with a disease duration of 6 to 10 years $(64 \%)$. This is similar in the 11 to 20 and 21 or

Table 9. Patient Distribution by Clinical Case Scenario and Group ( $n=108)$

\begin{tabular}{|c|c|c|c|c|c|c|}
\hline Group & CCS & Tophi & & Symptom Interval & CCS Frequency (\%) & Group Frequency (\%) \\
\hline \multirow{3}{*}{ A } & 1 & \multirow{3}{*}{ None } & \multirow{3}{*}{ Intermittent } & 1 attack in the past year & $17(15.74)$ & \multirow{3}{*}{$41(37.96)$} \\
\hline & 2 & & & 2-6 attacks in the past year & $16(14.81)$ & \\
\hline & 3 & & & $\geq 7$ attacks in the past year & $8(7.41)$ & \\
\hline \multirow{3}{*}{ B } & 4 & \multirow{3}{*}{ Stable } & \multirow{3}{*}{ Intermittent } & 1 attack in the past year & 11 (10.19) & \multirow{3}{*}{$33(30.56)$} \\
\hline & 5 & & & 2-6 attacks in the past year & $13(12.04)$ & \\
\hline & 6 & & & $\geq 7$ attacks in the past year & $9(8.33)$ & \\
\hline \multirow{4}{*}{ C } & 7 & \multirow{3}{*}{ Stable } & \multirow{3}{*}{ Chronic* } & Stable tophi in 1 joint & \multirow{4}{*}{\begin{tabular}{c}
\multicolumn{2}{c}{0} \\
$3(2.78)$ \\
$7(6.48)$ \\
$24(22.22)$
\end{tabular}} & \multirow{4}{*}{$34(31.48)$} \\
\hline & 8 & & & Stable tophi in $2-4$ joints & & \\
\hline & \multirow{2}{*}{9} & & & Stable tophi in $\geq 5$ joints & & \\
\hline & & Unstable** & & & & \\
\hline
\end{tabular}

${ }^{*}$ Persistent symptoms lasting 6 or more weeks

${ }^{* *}$ Could be one or more of the following: drainage, destructive effects/deformity, high risk for infection, rapid growth, severe inflammation

Table 10. Patient Distribution by Clinical Case Scenario and Group ( $n=108)$

\begin{tabular}{|c|c|c|c|c|c|c|c|c|c|}
\hline Group & CCS & Tophi & Symptom Interval & \multicolumn{2}{|c|}{ Tophi Frequency $(n=108)$} & \multicolumn{2}{|c|}{ Tophi Chronicity (n= 67) } & \multicolumn{2}{|c|}{ Tophi Stability $(n=67)$} \\
\hline A & $\begin{array}{l}1 \\
2 \\
3\end{array}$ & None & Intermittent & No Tophi & $41(37.96)$ & - & - & - & - \\
\hline B & $\begin{array}{l}4 \\
5 \\
6\end{array}$ & Stable & Intermittent & \multirow{3}{*}{ Tophi } & \multirow{3}{*}{$67(62.04)$} & Intermittent & $33(49.25)$ & \multirow{2}{*}{ Stable } & \multirow{2}{*}{$43(64.18)$} \\
\hline \multirow[t]{2}{*}{$\mathrm{C}$} & $\begin{array}{l}7 \\
8 \\
9\end{array}$ & $\begin{array}{c}\text { Stable } \\
\text { Stable }\end{array}$ & $\begin{array}{l}\text { Chronic* } \\
\text { Chronic* }^{*}\end{array}$ & & & \multirow[t]{2}{*}{ Chronic* } & \multirow[t]{2}{*}{$34(50.75)$} & & \\
\hline & 9 & Unstable** & Chronic* & & & & & Jnstable* & $24(35.82)$ \\
\hline
\end{tabular}

${ }^{*}$ Persistent symptoms lasting 6 or more weeks

${ }^{* *}$ Could be one or more of the following: drainage, destructive effects/deformity, high risk for infection, rapid growth, severe inflammation 
more groups with $68 \%$ and $76 \%$ prevalence. In comparing the prevalence of gout tophi stability among the different time sets $-31 \%$ of patients had unstable tophi in the 21 or more years. The five or less and 11 to 20 years' group had similar prevalence at $17 \%$ and $18 \%$, respectively. The identical majority in the two groups point to more factors involved in the stability of tophi and are not limited to deposition of MSU crystals as a function of time.

Comparing the population by tophi (Table 12), there was no significant difference in the two groups in terms of their age, time periods, and the number of initial joints involved. The number of initial joint involvement was not predictive of the development of tophi. The time to develop tophi was not measured, but the time from the appearance of symptoms to current CCS with tophi was bimodal at 6 and 21 years with a median of 15 years. There was also no difference in comorbidity prevalence between the tophi and

Table 11. Prevalence of Tophi Formation and Disease Duration

\begin{tabular}{cccccccc}
\multirow{2}{*}{$\begin{array}{c}\text { Disease } \\
\text { duration } \\
\text { (years) }\end{array}$} & \multirow{2}{*}{$\begin{array}{c}\text { No. of } \\
\text { patients }\end{array}$} & \multicolumn{2}{c}{ Cases with tophi } & & \multicolumn{2}{c}{$\begin{array}{c}\text { Cases with } \\
\text { unstable* tophi }\end{array}$} \\
\cline { 8 - 8 } \cline { 6 - 7 } & & No. & Percent & & No. & Percent \\
\hline or less & 29 & 12 & 41.38 & & 5 & 17.24 \\
6 to 10 & 22 & 14 & 63.64 & & 5 & 22.73 \\
11 to 20 & 28 & 19 & 67.86 & & 5 & 17.86 \\
21 or more & 29 & 22 & 75.86 & & 9 & 31.03 \\
\hline Total & 108 & 67 & 62.04 & & 24 & 22.22 \\
\hline
\end{tabular}

${ }^{*}$ Could be one or more of the following: drainage, destructive effects/ deformity, high risk for infection, rapid growth, severe inflammation. non-tophi groups. Chronic kidney disease, coronary heart disease, and stroke prevalence were higher in the "Chronic + Unstable" versus "Intermittent" group and the "Unstable" versus "Stable" group.

Upon the first consultation in the study, a median of 30.5 days had elapsed since the last flare of patients (Table 13). Thirty-four patients (31\%) reported that complete resolution of flare symptoms was spontaneous, and 74 (69\%) by medication. Patients recalled that flares would last from 1 to up to 30 days with a reported median of 3 days for patients who medicated and 4.5 days for patients with spontaneous resolution. These are typical of gout attacks which are characterized as rapidly developing synovitis in peripheral joints that are extremely painful but self-limiting, within several days or $1-2$ weeks. ${ }^{9}$

A clinical diagnosis was deemed sufficient for 9 out of 10 patients (Table 13). Five patients (5\%) underwent crystal analysis as a supplement for diagnosis. Before consult, colchicine (62\%) was the most common medication taken by patients, followed by oral NSAIDs (44\%). Medication was most often prescribed by Rheumatologists (61\%), followed by Internists (14\%). Compliance with gout medication was reported by $65 \%$. Ten patients self-medicated (non-medical practitioners) (9\%) with NSAIDs, steroids, colchicine, and paracetamol (Table 14). One patient from the group was medicated with an unrecalled NSAID together with a steroid.

Almost all patients were managed on an outpatient basis (87\%) (Table 15). Colchicine (87\%), followed by allopurinol (45\%) and febuxostat (30\%), was the most commonly

Table 12. Comparison by Tophi

\begin{tabular}{|c|c|c|c|c|c|c|c|c|c|c|c|c|}
\hline & \multicolumn{4}{|c|}{ Current Age } & \multicolumn{4}{|c|}{ Age at First Symptoms } & \multicolumn{4}{|c|}{ Time First Symptoms to Current CCS } \\
\hline & Mode & Median & Range & Mean + SD & Mode & Median & Range & Mean + SD & Mode & Median & Range & Mean + SD \\
\hline Gout Patients ( $n=108)$ & 58 & 61 & $26-80$ & $55.31 \pm 11.88$ & 41 & 40 & $15-72$ & $41.91 \pm 12.88$ & 11 & 1 & $0-50$ & $13.39 \pm 10.46$ \\
\hline No Tophi $(n=41)$ & 61 & 62 & $26-75$ & $56.32 \pm 12.62$ & 48 & 55 & $15-68$ & $45.46 \pm 13.03$ & 8 & 0 & $0-40$ & 10.56 \\
\hline Tophi $(n=67)$ & 57 & 61 & $30-80$ & 54.65 & 40 & 40 & $19-72$ & 39.67 & 15 & 6 & $1-50$ & .23 \\
\hline Intermittent $(\mathrm{n}=33)$ & 59 & 51 & $34-80$ & $58.76 \pm 10.65$ & 41 & 50 & $19-72$ & $43.39 \pm 12.57$ & 16 & 16 & $1-50$ & 0.41 \\
\hline Chronic + Unstable ${ }^{* *}(n=34)$ & 51 & 61 & $30-73$ & $50.74 \pm 10.82$ & 35.5 & 25 & $19-57$ & $36.06 \pm 11.02$ & 14 & 7 & $1-36$ & $14.68 \pm 9.88$ \\
\hline Stable $(n=43)$ & 59 & 59 & $32-80$ & $56.21 \pm 11.19$ & 40 & 30 & $19-72$ & $41.07 \pm 12.53$ & 16 & 6 & $1-50$ & $15.14 \pm 10.09$ \\
\hline Unstable $^{* *}(\mathrm{n}=24)$ & 54 & 61 & $30-73$ & $51.96 \pm 11.44$ & 40 & 25 & $19-57$ & $37.17 \pm 11.64$ & 14 & 21 & $1-36$ & $14.79 \pm 10.25$ \\
\hline
\end{tabular}

${ }^{*}$ Persistent symptoms lasting 6 or more weeks

${ }^{* *}$ Could be one or more of the following: drainage, destructive effects/deformity, high risk for infection, rapid growth, severe inflammation SD - Standard Deviation

\begin{tabular}{|c|c|c|c|c|c|c|c|c|}
\hline & \multicolumn{3}{|c|}{ Initial Joint Involvement Number } & \multicolumn{5}{|c|}{ Comorbidity } \\
\hline & 1 Joint & 2-3 Joints & $\geq 4$ Joints & HPN & CKD & DM & CHD & Stroke \\
\hline Gout Patients $(n=108)$ & $58(53.70)$ & $45(41.67)$ & $5(4.63)$ & $46(42.59)$ & $36(33.33)$ & $17(15.74)$ & $15(13.89)$ & $4(3.70)$ \\
\hline No Tophi $(n=41)$ & $22(53.66)$ & $16(39.02)$ & $3(7.32)$ & $19(46.34)$ & $11(26.83)$ & $6(14.63)$ & $8(19.51)$ & $1(2.44)$ \\
\hline Tophi $(n=67)$ & $36(53.73)$ & $29(43.28)$ & $2(2.99)$ & $27(40.30)$ & 25 (37.31) & $11(16.42)$ & $7(10.45)$ & $3(4.48)$ \\
\hline Intermittent $(\mathrm{n}=33)$ & $16(48.48)$ & $15(45.45)$ & $2(6.06)$ & $13(39.39)$ & $8(24.24)$ & $5(15.15)$ & $2(6.06)$ & 1 (3.03) \\
\hline Chronic + Unstable ${ }^{* *}(n=34)$ & $20(58.82)$ & $14(41.18)$ & 0 & $14(41.18)$ & $17(50)$ & $6(17.65)$ & $5(14.71)$ & $2(5.88)$ \\
\hline Stable $(n=43)$ & $22(51.16)$ & 19 (44.19) & $2(4.65)$ & $18(41.86)$ & $12(27.91)$ & $7(16.28)$ & $3(6.98)$ & 1 (2.33) \\
\hline Unstable $^{* *}(\mathrm{n}=24)$ & $14(58.33)$ & $10(41.67)$ & 0 & 9 (37.5) & $13(54.17)$ & $4(16.67)$ & $4(16.67)$ & $2(8.33)$ \\
\hline
\end{tabular}

*Persistent symptoms lasting 6 or more weeks

${ }^{* *}$ Could be one or more of the following: drainage, destructive effects/deformity, high risk for infection, rapid growth, severe inflammation HPN - Hypertension; CKD - Chronic Kidney Disease; DM - Diabetes Mellitus; CHD - Coronary Heart Disease 
Table 13. First Consultation Findings $(n=108)$

\begin{tabular}{|c|c|}
\hline & Frequency (\%); Median (Range) \\
\hline Days from previous flare & $30.5(0-1,835)$ \\
\hline \multicolumn{2}{|l|}{ Flare resolution } \\
\hline Spontaneous & $34(31.48)$ \\
\hline Medicated & $74(68.52)$ \\
\hline Days for flare resolution & $3(1-30)$ \\
\hline Spontaneous & $4.5(1-21)$ \\
\hline Medicated & $3(1-30)$ \\
\hline \multicolumn{2}{|l|}{ Diagnostic modality } \\
\hline Clinical & 98 (90.74) \\
\hline Crystal & $5(4.63)$ \\
\hline Ultrasound & 0 \\
\hline X-ray & 0 \\
\hline Unreported & $5(4.63)$ \\
\hline \multicolumn{2}{|l|}{ Medicines taken prior to consult } \\
\hline Colchicine & $67(62.04)$ \\
\hline Oral NSAIDs & $48(44.44)$ \\
\hline Allopurinol & $31(28.70)$ \\
\hline Febuxostat & $26(24.07)$ \\
\hline Steroid & $22(20.37)$ \\
\hline Paracetamol & $10(9.26)$ \\
\hline Tramadol & 8 (7.41) \\
\hline Herbal & $4(3.70)$ \\
\hline Tramadol + Paracetamol & 2 (1.85) \\
\hline Topical NSAIDs & $1(0.93)$ \\
\hline \multicolumn{2}{|l|}{ Prescriptionist } \\
\hline Rheumatologist & $66(61.11)$ \\
\hline Internal Medicine & 15 (13.89) \\
\hline Non-medical practitioner & $10(9.26)$ \\
\hline General practitioner & 8 (7.41) \\
\hline Surgeon/Orthopedist & $5(4.63)$ \\
\hline Family medical doctor & 2 (1.85) \\
\hline Obstetrician-gynecologist & $1(0.93)$ \\
\hline Unrecalled & $4(3.70)$ \\
\hline Compliance to medication & $70(64.81)$ \\
\hline \multicolumn{2}{|l|}{ Laboratory } \\
\hline Hemoglobin (g/L) [n=51] & $130(15.7-177)$ \\
\hline Hematocrit [n=52] & $0.4(0.21-46.1)$ \\
\hline WBC (K/uL) [n=52] & $8.3(2.6-29.4)$ \\
\hline Platelets (K/uL) [n=47] & $280(92-746)$ \\
\hline ALT (IU/L) [n=25] & $36(10.47-182)$ \\
\hline AST (IU/L) [n=21] & $39.3(14.23-195)$ \\
\hline FBS (mmol/L) [n=19] & $5.44(4.22-13.06)$ \\
\hline HBA1c [n=4] & $8.37 \pm 1.6$ \\
\hline $\begin{array}{l}\text { Total cholesterol }(\mathrm{mmol} / \mathrm{L}) \\
{[\mathrm{n}=21]}\end{array}$ & $5.27(2.95-12.05)$ \\
\hline Triglycerides (mmol/L) [n=18] & $1.51 \pm 0.55$ \\
\hline $\mathrm{HDL}(\mathrm{mmol} / \mathrm{L})[\mathrm{n}=19]$ & $1.24(0.465-7.37)$ \\
\hline LDL (mmol/L) [n=19] & $2.94 \pm 1.01$ \\
\hline Creatinine (umol/L) [n=73] & $118(41-1617)$ \\
\hline No tophi [n=24] & $\begin{array}{c}118.9(59-1617) \\
208.99 \pm 316.93\end{array}$ \\
\hline Tophi [n=49] & 118 (41-845); $156.8 \pm 124.02$ \\
\hline Uric acid (mg/dL) [n=77] & $7.99 \pm 3.3$ \\
\hline No tophi [24] & 7.11 (1.02-15.6); 7.24 2.97 \\
\hline Tophi [n=49] & 8.14 (0.41-18.4); $8.42 \pm 3.43$ \\
\hline
\end{tabular}

Table 14. Non-Medical Practitioner Medication $(n=10)$

\begin{tabular}{lc} 
& Frequency (\%) \\
Unrecalled NSAIDs & $6(60 \%)$ \\
Unrecalled NSAIDs + Steroid & $1(10 \%)$ \\
Steroid + Colchicine & $1(10 \%)$ \\
Colchicine + Paracetamol & $1(10 \%)$ \\
Herbal & $1(10 \%)$ \\
\hline
\end{tabular}

Table 15. Overall Medical Course $(n=108)$

\begin{tabular}{lclc} 
& \multicolumn{3}{c}{ Frequency (\%) } \\
\cline { 2 - 4 } Management & Outpatient & Admitted & Total \\
\hline Treatment during consult/referral & $87.04)$ & $14(12.96)$ \\
Colchicine & $81(86.17)$ & $13(92.86)$ & $94(87.04)$ \\
Allopurinol & $45(47.87)$ & $4(28.57)$ & $49(45.37)$ \\
Febuxostat & $28(29.79)$ & $4(28.57)$ & $32(29.63)$ \\
Oral NSAIDs & $14(14.89)$ & $1(7.14)$ & $15(13.89)$ \\
Oral steroids & $9(9.57)$ & $6(42.86)$ & $15(13.89)$ \\
Tramadol + Paracetamol & $10(10.64)$ & 0 & $10(9.26)$ \\
Paracetamol & $8(8.51)$ & $1(7.14)$ & $9(8.33)$ \\
Tramadol & $8(8.51)$ & 0 & $8(7.41)$ \\
Losartan & $8(8.51)$ & 0 & $8(7.41)$ \\
Arthrocentesis & $3(3.19)$ & $2(14.29)$ & $5(4.63)$ \\
Intravenous steroids & $1(1.06)$ & $4(28.57)$ & $5(4.63)$ \\
Intra-articular steroids & $2(2.13)$ & 0 & $2(1.85)$ \\
Topical NSAIDs & $1(1.06)$ & 0 & $1(0.93)$ \\
Fenofibrate & 0 & 0 & 0 \\
Others & $1(1.06)$ & 0 & $1(1.06)$ \\
\hline Education & $16(17.02)$ & $1(7.14)$ & $17(15.74)$ \\
\hline
\end{tabular}

NSAID, a nonsteroidal anti-inflammatory drug

prescribed anti-gout medication. Only 17 patients (16\%) overall received education for gout during their consultation.

Thirty-eight patients (35\%) were diagnosed with an ongoing gout flare during the first consultation (Table 16). The majority of these (42\%) had one or two large joints involved. Pain grade was mainly moderate to severe, and patients tended to consult late (more than 36 hours) for their ongoing gout attack (71\%).

Most patients with an ongoing gout flare were treated on an outpatient basis (71\%) (Table 17). Only four patients underwent arthrocentesis (11\%), and two patients received intra-articular steroids (5\%). Nearly all patients were prescribed colchicine (92\%). The most common antiinflammatory medication given was oral steroids (32\%) followed by oral NSAIDs (21\%). Urate lowering agents, allopurinol (44\%), and febuxostat (24\%) were also given.

Patients without a gout flare were mainly treated on an outpatient basis (96\%) (Table 18). Only one patient underwent arthrocentesis (1\%). Nearly all patients were prescribed colchicine (84\%). The most common anti-inflammatory medication given was oral NSAIDs $(10 \%)$ followed by oral steroids (4\%). Urate lowering agents, allopurinol (46\%), and febuxostat (33\%) were also given. 
Only 19 patients had a first follow-up visit, all outpatient (Table 19). The median duration from the first consult was $84(3-325)$ days. Gout flare in one or two large joints was ongoing for three patients. It was noted that all three patients with ongoing flare were all newly diagnosed cases of gout. All three were seen previously outpatient and received no gout education. Two were treated with oral NSAIDs and colchicine, and one was treated with colchicine only. None were given urate-lowering medication. Only two patients claimed to be compliant with their medication but followed up only after 21 to 91 days due to repeat gouty flare pain.

CCS 1 to 5 were predominated by patients with age at symptom onset of 40 years and older (Table 20). On

Table 16. Gouty Flare on First Consult $(n=38)$

\begin{tabular}{lr}
\hline & Frequency (\%) \\
\hline Involved joints & \\
1 or few small & $10(26.32)$ \\
1-2 large* & $16(42.11)$ \\
Polyarticular** & $12(31.58)$ \\
\hline Pain (Visual Analog Scale 1 - 10) & \\
$\quad$ Mild <5 & $9(23.68)$ \\
Moderate 5-6 & $13(34.21)$ \\
Severe >6 & $12(31.58)$ \\
Not Reported & $4(10.53)$ \\
\hline Duration of pain (hours) & \\
$\quad$ Early <12 & $4(10.53)$ \\
Well Established 12-36 & $7(18.42)$ \\
Late $>36$ & $27(71.05)$ \\
\hline
\end{tabular}

*Large - ankle, knee, wrist, elbow, hip, shoulder

${ }^{* *} 4$ or more joints, with arthritis involving more than one region [forefoot (MTP, toes), midfoot (tarsal), ankle/hindfoot, knee, hip, fingers, wrist, elbow, shoulder, other]. An attack involving three separate large joints is considered a form of polyarticular gout.

Table 17. Medical Course of Patients with Gout Flare $(n=38)$

\begin{tabular}{llll} 
& \multicolumn{3}{c}{ Frequency (\%) } \\
\cline { 2 - 4 } Management & Outpatient & Admitted & Total \\
\hline Treatment during consult/referral & $27(71.05)$ & $11(28.95)$ \\
Colchicine & $25(92.59)$ & $10(90.91)$ & $35(92.11)$ \\
Allopurinol & $15(55.56)$ & $2(18.19)$ & $17(44.47)$ \\
Febuxostat & $6(22.22)$ & $3(27.27)$ & $9(23.68)$ \\
Oral NSAIDs & $7(25.93)$ & $1(9.09)$ & $8(21.05)$ \\
Oral steroids & $7(25.93)$ & $5(45.45)$ & $12(31.58)$ \\
Tramadol + Paracetamol & $4(14.81)$ & 0 & $4(10.53)$ \\
Paracetamol & $1(3.7)$ & 0 & $1(2.63)$ \\
Tramadol & $3(11.11)$ & 0 & $3(7.89)$ \\
Losartan & $2(7.41)$ & 0 & $2(5.26)$ \\
Arthrocentesis & $3(11.11)$ & $1(9.09)$ & $4(10.53)$ \\
Intravenous steroids & $1(3.7)$ & $4(36.36)$ & $5(13.16)$ \\
Intra-articular steroids & $2(7.41)$ & 0 & $2(5.26)$ \\
Topical NSAIDs & 0 & 0 & 0 \\
Fenofibrate & 0 & 0 & 0 \\
Others & $1(3.7)$ & 0 & $1(2.63)$ \\
\hline Education & $4(14.81)$ & $1(9.09)$ & $5(13.16)$ \\
\hline
\end{tabular}

NSAID, a nonsteroidal anti-inflammatory drug the other hand, younger-onset was more frequent with the higher clinical scenario stages - CCS 6 to 9 (56\%). At least half of the patients at stages 1,3 , and 4 were hypertensive. There was a low prevalence of obesity across all CCS; and, there were only two patients with metabolic syndrome in the whole study - both in CCS 4 with low prevalence.

\section{DISCUSSION}

\section{Patient Characteristics}

Gout is a crystal deposition disease that arises when supersaturation of body tissues with urate occurs, leading to the formation of MSU crystals in and around joints. ${ }^{2}$ It is the most prevalent inflammatory arthritis in men and is associated with impaired quality of life. ${ }^{21}$ In western

Table 18. Medical Course of Patients without Gout Flare $(n=70)$

\begin{tabular}{llll} 
& \multicolumn{3}{c}{ Frequency (\%) } \\
\cline { 2 - 3 } & Outpatient & Admitted & Total \\
\hline Management & $67(95.71)$ & $3(4.29)$ \\
\hline Treatment during consult/referral & & \\
Colchicine & $56(83.58)$ & $3(100)$ & $59(84.29)$ \\
Allopurinol & $30(44.78)$ & $2(66.67)$ & $32(45.71)$ \\
Febuxostat & $22(32.84)$ & $1(33.33)$ & $23(32.86)$ \\
Oral NSAIDs & $7(10.45)$ & 0 & $7(10)$ \\
Oral steroids & $2(2.99)$ & $1(33.33)$ & $3(4.29)$ \\
Tramadol + Paracetamol & $6(8.96)$ & 0 & $6(8.57)$ \\
Paracetamol & $7(10.45)$ & $1(33.33)$ & $8(11.43)$ \\
Tramadol & $5(7.46)$ & 0 & $5(7.14)$ \\
Losartan & $6(8.96)$ & 0 & $6(8.57)$ \\
Arthrocentesis & 0 & $1(33.33)$ & $1(1.43)$ \\
Intravenous steroids & 0 & 0 & 0 \\
Intra-articular steroids & 0 & 0 & 0 \\
Topical NSAIDs & $1(1.49)$ & 0 & $1(1.43)$ \\
Fenofibrate & 0 & 0 & 0 \\
Others & 0 & 0 & 0 \\
\hline Education & $12(17.91)$ & 0 & $12(17.14)$ \\
\hline
\end{tabular}

NSAID, a nonsteroidal anti-inflammatory drug

Table 19. First follow-up $(n=19)$

\begin{tabular}{lc} 
& Frequency (\%); Median (Range) \\
\hline Seen outpatient & $19(100)$ \\
\hline Days from last flare & $40(7-500)$ \\
Flare on first consult $(\mathrm{n}=9)$ & $21(7-90)$ \\
No flare on first consult $(\mathrm{n}=10)$ & $183(7-500)$ \\
\hline Days from previous consult & $84(3-325)$ \\
Flare on first consult $(\mathrm{n}=9)$ & $50(7-325)$ \\
No flare on first consult $(\mathrm{n}=10)$ & $84(3-108)$ \\
\hline Resolution & \\
Medicated & $18(94.74)$ \\
Spontaneous & $1(5.26)$ \\
\hline Currently in gout attack & $3(15.79)$ \\
1-2 large joints [n=3] & $3(100)$ \\
\hline
\end{tabular}


Table 20. Distribution of Factors for Gout Attacks across Clinical Case Scenario

\begin{tabular}{|c|c|c|c|c|c|c|c|c|c|}
\hline & $\begin{array}{c}1 \\
(n=17)\end{array}$ & $\begin{array}{c}2 \\
(n=16)\end{array}$ & $\begin{array}{c}3 \\
(n=8)\end{array}$ & $\begin{array}{c}4 \\
(n=11)\end{array}$ & $\begin{array}{c}5 \\
(n=13)\end{array}$ & $\begin{array}{c}6 \\
(n=9)\end{array}$ & $\begin{array}{c}7 \\
(n=0)\end{array}$ & $\begin{array}{c}8 \\
(n=3)\end{array}$ & $\begin{array}{c}9 \\
(n=31)\end{array}$ \\
\hline & \multicolumn{9}{|c|}{ Frequency (\%) } \\
\hline \multicolumn{10}{|l|}{ Age at symptom onset, years } \\
\hline$<40$ & $4(23.53)$ & $6(37.50)$ & $2(25)$ & 2 (18.18) & $4(30.77)$ & $6(66.67)$ & 0 & $1(33.33)$ & $17(54.84)$ \\
\hline$\geq 40$ & $13(76.47)$ & $10(62.50)$ & $6(75)$ & $9(81.82)$ & $9(69.23)$ & $3(33.33)$ & 0 & $2(66.67)$ & $14(45.16)$ \\
\hline Male & $16(94.12)$ & $16(100)$ & $8(100)$ & $15(100)$ & $15(100)$ & $9(100)$ & 0 & $3(100)$ & $30(96.77)$ \\
\hline Hypertension & $9(52.94)$ & $6(37.50)$ & $4(50)$ & $6(54.54)$ & $4(26.67)$ & $3(33.33)$ & 0 & $1(33.33)$ & $13(41.93)$ \\
\hline \multicolumn{10}{|l|}{ BMI } \\
\hline Underweight & $1(5.88)$ & $1(6.25)$ & $1(12.50)$ & $1(9.09)$ & 0 & $1(11.11)$ & 0 & 0 & $5(16.13)$ \\
\hline Normal & $6(35.29)$ & $10(62.50)$ & $4(50)$ & $9(81.82)$ & $4(30.77)$ & $5(55.66)$ & 0 & $2(66.67)$ & $11(35.48)$ \\
\hline Overweight & $8(47.06)$ & $4(25)$ & $1(12.50)$ & $1(9.09)$ & $7(53.85)$ & $1(6.67)$ & 0 & $1(33.33)$ & 12 (38.71) \\
\hline Obese & $2(11.76)$ & $1(6.25)$ & $2(25)$ & 0 & $2(15.38)$ & $2(22.22)$ & 0 & 0 & $3(9.68)$ \\
\hline Metabolic Syndrome & 0 & 0 & 0 & $2(18.18)$ & 0 & 0 & 0 & 0 & 0 \\
\hline Uric acid level (mg/dL) & $\begin{array}{c}\mathrm{n}=14] \\
6.96 \pm 2.06\end{array}$ & $\begin{array}{c}{[n=8]} \\
8.63 \pm 4.35\end{array}$ & $\begin{array}{c}{[n=6]} \\
6.04 \pm 2.26\end{array}$ & $\begin{array}{c}{[n=7]} \\
6.74 \pm 2.12\end{array}$ & $\begin{array}{c}{[n=11]} \\
8.02 \pm 4.52\end{array}$ & $\begin{array}{c}{[n=4]} \\
8.23 \pm 3.38\end{array}$ & 0 & $\begin{array}{c}n=3] \\
6.9 \pm 1.15\end{array}$ & $\begin{array}{c}{[n=24]} \\
9.31 \pm 3.29\end{array}$ \\
\hline Nephrolithiasis & $1(5.88)$ & $1(6.25)$ & 0 & $1(9.09)$ & $2(15.38)$ & 0 & 0 & 0 & $1(4)$ \\
\hline
\end{tabular}

countries, the general prevalence of gout is $1-4 \%$ of the general population; ${ }^{22}$ in the Philippines, it has an overall prevalence of $1.6 \%$ in the population. ${ }^{3,4}$ Gout occurs in $3-6 \%$ in men and $1-2 \%$ in women. ${ }^{6}$ In the study, only two patients, were female while the rest were male. Both females had chronic kidney disease, menopause, and started experiencing gout symptoms at 55 years old. This is typical for female patients with gout who develop increased serum uric acid when they lose estrogen protection during menopause.

Patients with gout are more likely to have certain sociodemographic and lifestyle variables like older age, low socioeconomic status, increased body mass index, and heavy alcohol consumption. ${ }^{23}$ Exposure to diuretics, renal disease, and the proportion of patients who underwent transplantation were also more common in gout patients at diagnosis compared to the general population. All cardiovascular diseases were common and highly associated with gout at gout diagnosis, with hypertension being the most frequent. Recent studies also identified renal calculi in a third of patients with gout. ${ }^{13}$ Race was also seen to play a role in developing gout. Filipinos, in particular, are at a higher risk of elevated uric acid levels and gout; comorbidities including obesity, hypertension, diabetes, renal impairment, and heart disease were also common in this population. ${ }^{24}$

While comorbidities are more prevalent in hyperuricemia, they are linked to increased gout risk and secondary complications. ${ }^{9}$ There is evidence that obesity as part of the metabolic syndrome consistently increases the risk of gout - obese women had a 2.4-fold greater risk of developing gout than non-obese women; early adult obesity in women was associated with a 2.8-fold increased risk of gout compared with non-obese women. ${ }^{25}$ Hypertension is an independent risk factor for gout with a hazard ratio of $2 ;^{26}$ and, chronic kidney disease was associated with a hazard ratio of 1.61 for increased gout risk. ${ }^{27}$
The demographic characteristics of the patients included in this study reflect most of these data; The most commonly identified comorbidities were hypertension (43\%), chronic kidney disease (33\%), and diabetes mellitus (16\%); 6 had renal stones (6\%), and most were alcohol beverage drinkers (60\%). Of interest, only a small proportion of subjects were obese (10\%), and an even smaller portion had metabolic syndrome (2\%). While western data show that cigarette smoking might confer protection from developing gout, ${ }^{24}$ most of the patients in this study were smokers (53\%).

Gout is a risk factor for cardiovascular disease, which encompasses coronary artery disease, ischemic cerebrovascular stroke, and peripheral arterial disease. ${ }^{28}$ Seminog et al. reviewed datasets spanning from 1963 to 1998 and 1999 to 2011, which revealed an increased risk of myocardial infarction (RR 1.82) and stroke (RR 1.71). ${ }^{29} \mathrm{~A}$ meta-analysis in 2015 by Liu et al. showed similar results for an increased risk of myocardial infarction observed in both women (RR 1.62) and men (RR 1.45) and increased risk in gout patients with younger age of onset. ${ }^{30}$

In this study, patients with stroke and coronary heart disease were identified. Four patients had a history of stroke (4\%) with hypertension in 3 patients and chronic kidney disease in 3 patients (Figure 1). None of them had associated diabetes or metabolic syndrome. Fifteen patients had coronary heart disease (14\%) with chronic kidney disease in 9 patients and hypertension, dyslipidemia, and diabetes in 8,3 , and 2 patients, respectively (Figure 2). Three patients with coronary heart disease had no other comorbidity. None of them had metabolic syndrome. The two figures show the associated comorbidities in these groups. It is noted that gout with hypertension and chronic kidney disease are more prevalent in stroke and coronary heart disease. The complexity of disease overlap, treatment, and ever-changing environmental factors make discernment of gout's role in its complications difficult. It also raises the question - does 


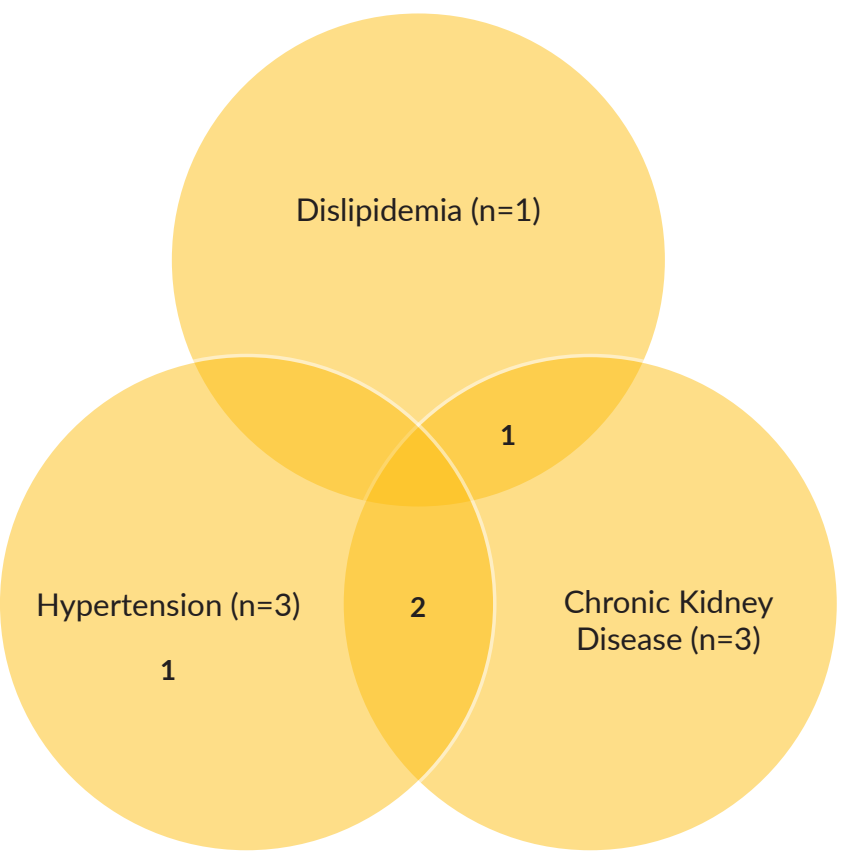

Figure 1. Gout Patients with Stroke (Total = 4).

treating gout improve cardiovascular disease outcomes? More patients and further studies are needed to explore the intricate relationships between gout, its comorbidities, and Filipino complications.

\section{Comparison of Philippine Clinical Profile}

The clinical profile of gout in the Philippines was investigated in one of the first local scarce studies of gout. ${ }^{31}$ In 1975, Torralba et al. conducted a review with 260 patients with gout from a private clinic and a tertiary hospital at the University of Santo Tomas, Philippines.

The mean age at first symptoms in the study was 47 years in the males and 64 years in the females (Table 21). The new cohort's mean age was earlier -42 years in males and 55 years old in females. Comparing the age groups in males, the 1975 cohort had a predominance of patients

Table 21. Age at First Symptoms and Sex Distribution

\begin{tabular}{cccccc} 
Age & \multicolumn{2}{c}{2019} & & \multicolumn{2}{c}{1975} \\
\cline { 2 - 3 } \cline { 5 - 6 } Group & Males & Females & & Males & Females \\
$15-19$ & 3 & 0 & & 2 & 0 \\
$20-24$ & 6 & 0 & & 5 & 0 \\
$25-29$ & 9 & 0 & & 15 & 0 \\
$30-39$ & 24 & 0 & & 47 & 0 \\
$40-49$ & 33 & 0 & & 61 & 3 \\
$50-59$ & 19 & 2 & & 64 & 0 \\
$60-69$ & 9 & 0 & & 44 & 4 \\
$70-79$ & 3 & 0 & & 12 & 1 \\
$80-89$ & 0 & 0 & & 0 & 2 \\
\hline Total & $106(98 \%)$ & $2(2 \%)$ & $250(96 \%)$ & $10(4 \%)$ \\
\hline
\end{tabular}

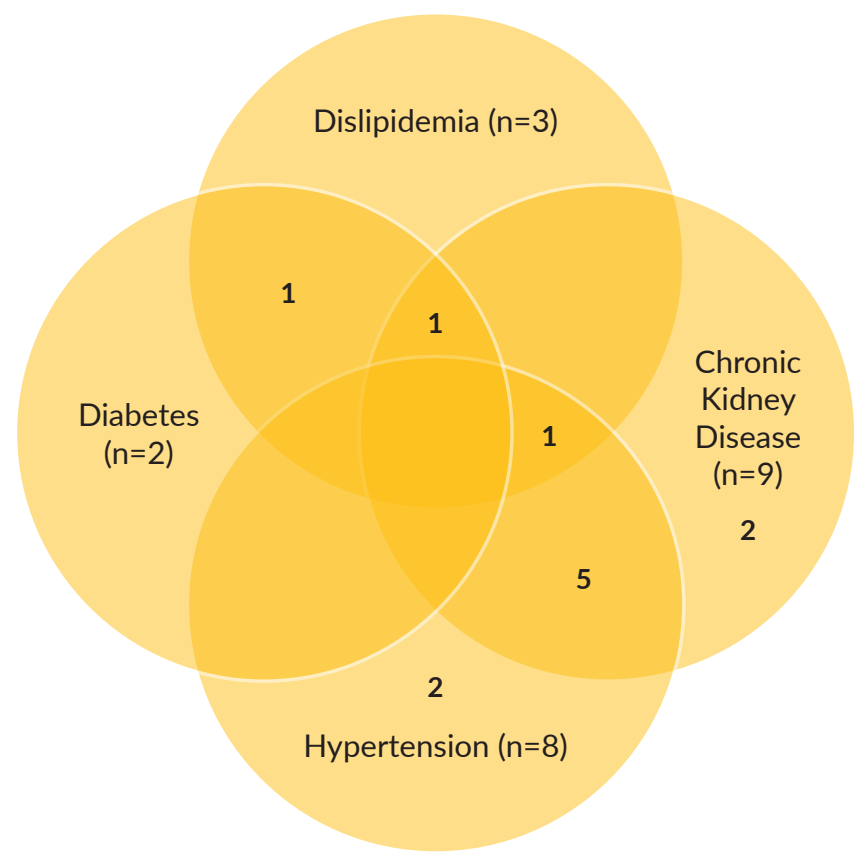

Figure 2. Gout Patients with Coronary Heart Disease (Total $=15)$.

in the "50 to 59" and "40 to 49" age group while the 2019 cohort had predominance in the " 40 to 49 " and "30 to 39 " age group. Sex distribution was not significantly different in both groups, with gout incidence in males at $96 \%$, females at $4 \%$ in the 1975 cohort and $98 \%$ in males, $2 \%$ in females in the 2019 cohort.

Figure 3 shows the differences in comorbidities between the 1975 and 2019 cohorts. Obesity is similar between the two groups, while coronary heart disease and stroke, hypertension, diabetes, and chronic kidney disease are more prevalent now than 40 years ago. Urolithiasis was less prevalent in the new cohort.

This comparison shows a decrease in the age of early gout manifestations in the 2019 cohort. This may translate to an earlier incidence of gout in the Philippines. This is multifactorial in origin, and increased comorbidity,

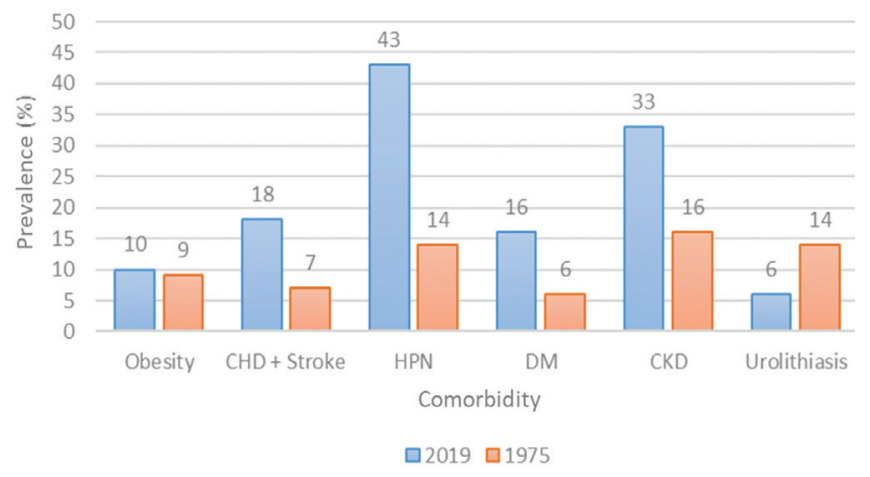

Figure 3. Comorbidity Prevalence Comparison. 


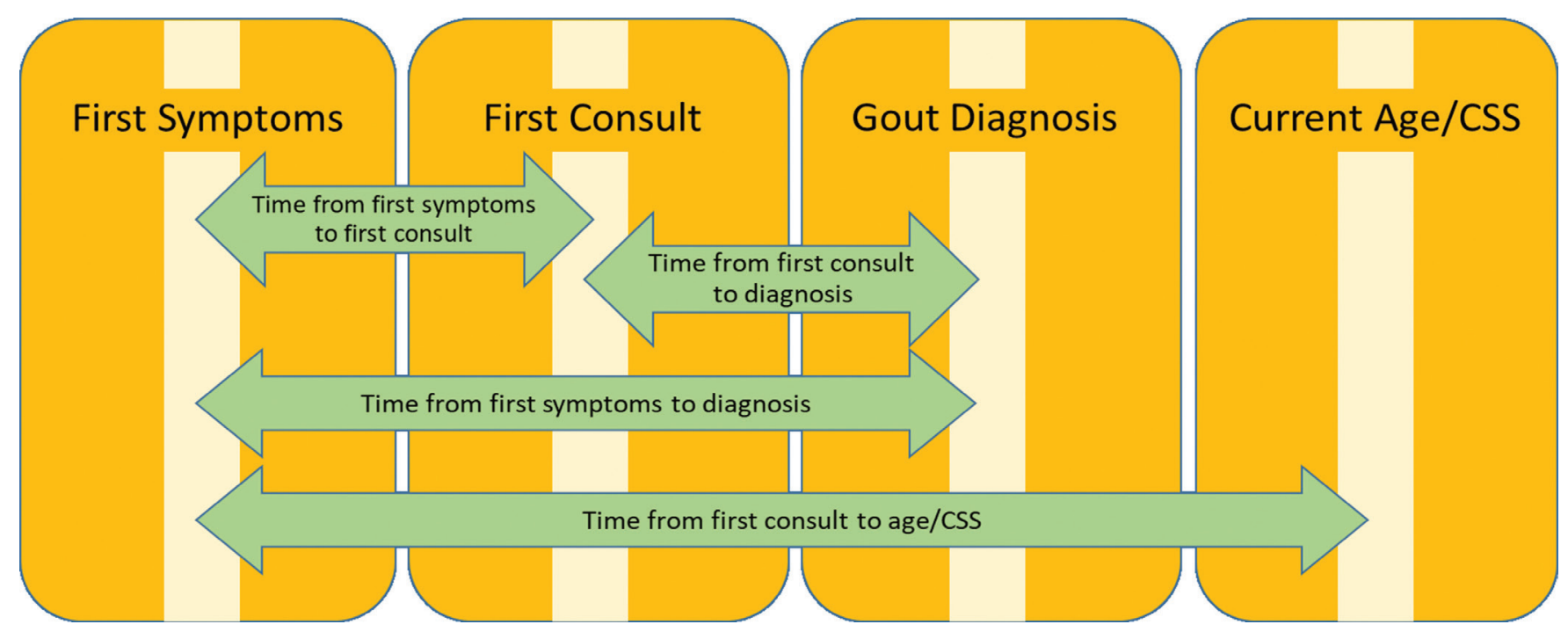

Figure 4. Timeline from Symptoms to CCS.

lifestyle changes, diet, patient education, and early access to healthcare are likely contributory.

\section{Clinical Presentation}

Gout undergoes four stages during its course, starting with asymptomatic hyperuricemia. Patients have no symptoms or signs and are usually incidentally discovered when measuring SUA (serum level greater than $7 \mathrm{mg} / \mathrm{dL}$ ). On the other hand, an acute gouty attack is characterized by monoarthritis episodes that peak within hours to severely inflamed joints with cardinal signs of inflammation, including redness, hotness, tenderness, swelling, and loss of function. These attacks have a predilection for lower extremities, such as the first MTP, which is the most typical acute gout in Western data. Other joints affected are the tarsal and metatarsal joints, ankles, knees, wrists, MCPs, and interphalangeal joints of the hands. ${ }^{5}$ In one of the first gout studies in Filipinos conducted by Torralba in 1975, the most common initial joint involved was the MTP (50\%), followed by the ankle (32\%), the knee (17\%), and the wrist (1\%). ${ }^{31}$ The new cohort is different, showing that the most common joint initially involved was the ankle (52\%), followed by the first MTP (40\%), the knee (30\%), and the midfoot (16\%). Monoarthritis was the most common gout presentation in this cohort, but surprisingly, almost half of patients presented with two or more joint involvement (46.3\%) at the onset. It is also pertinent to note that all patients presented with polyarticular joint pain (100\%) involved the ankle and first MTP. This suggests that gout may be highly considered in patients with polyarticular symptoms involving the ankle and MTP.

When the acute attack settles down within hours to days spontaneously or following the introduction of colchicine or NSAIDs, patients enter into remission or an intercritical phase, where any symptoms are absent. ${ }^{6,15}$ Untreated disease progresses to become chronic tophaceous gout where there is joint destruction and palpable tophi formation. ${ }^{6}$ In our cohort, patients are further classified according to the presence or absence of tophi, the chronicity of symptoms, and the number of joints involved. A majority on initial consultation already had tophi. Clinical scenario stages 1 to 3 , or those without tophi, were predominated by patients with age at symptom onset of 40 years and older (Table 20). On the other hand, younger-onset was more frequent with the higher clinical scenario stages (56\%). This is consistent with the finding that although patients in this cohort experience symptoms at age 41, the average age of diagnosis is more than seven years later.

When analyzing the timeline from a patient's first experience of gout symptoms, first consultation, and when they are finally diagnosed, one must be aware of the periods in between (Figure 4). These periods are not mutually exclusive and can overlap.

The period from first symptoms to the first consultation's age tells us the amount of time a patient takes to seek out consultation actively. This may represent how often a patient would tolerate his symptoms before consult and their perceived importance of medical consultation. It is during this time that a patient may experiment and selfmedicate with an alternative or unprescribed medication. This period is essential even if the proper diagnosis is not achieved immediately. The early consult may lead to the early prescription of anti-inflammatory medication, early referral to a specialist, or both.

The period from first symptoms to age at diagnosis tells us the amount of time a patient spent before being accurately diagnosed with gout. It is limited by the case difficulty, healthcare provider's knowledge on gout, and time to seek specialist care. It is inclusive of the time to consult and be accurately diagnosed after the first consultation. 
The first consult to diagnosis period includes the times a patient has been incorrectly diagnosed, lost to follow up, or both. The sooner a patient is diagnosed, the sooner they receive the proper medication and ULT started. A prolonged time from first symptoms to current CCS may reflect how far the CCS is due to lack or missed diagnosis, treatment, or both. Identifying the circumstances prolonging these periods is vital in creating targeted strategies by private and government health institutions. While education to laypersons may reduce the time lost to consultation, on physicians' side, improvement of the clinical eye or when to refer to a specialist must be touted.

Though there was no significant difference among CCS in periods, there is a more prolonged time in the higher CCS (Table 3) and later diagnosis (Table 4). This movement warrants that early identification and diagnosis of gout may be a factor for preventing elevated CCS. The data show that early symptom onset alone does not lead to the formation of unstable tophi or a higher CCS. Prolonged disease duration, as well as late diagnosis and treatment, contribute. When a patient presents with significant joint symptoms, immediate diagnosis or referral to a specialist will be beneficial. Patients also have to be compliant with their medication and follow up appropriately so that CCS does not worsen. The data gathered provides a benchmark for monitoring.

At least half of the patients at CCS 3 (those who have intermittent symptoms amounting to 7 or more episodes in a year) have hypertension. This is consistent with Rashid's earlier study, where patients with $\geq 3$ gout flares had a higher percentage of comorbidities, including hypertension, versus the control groups. ${ }^{32}$ Those patients also had more use of anti-hypertensives and diuretics versus patients without gout flares.

In this study, the diagnosis of gout was mainly made by rheumatologists rather than primary health care physicians. This is perhaps because the research was conducted in a tertiary care hospital. As in most countries, gout diagnosis was made clinically rather than by crystal analysis or ultrasound imaging. ${ }^{6}$ Furthermore, the high number of patients taking medicine without consulting their physician is problematic as it may lead to kidney or liver damage.

\section{Risk Factors for Flares}

In this cohort, thirty-eight patients were diagnosed with gout flare during the initial consultation (35\%). The majority of these had one or two large joints involved (42\%). The pain was primarily moderate to severe; and, patients tended to consult late (more than 36 hours) for their ongoing gout attack (71\%). This is significant as it represents the amount of time lost in starting medication which is best given within 24 hours of a gouty flare.

Eighty-eight patients reported $\geq 1$ gout flare triggers (81\%), with fifty-seven patients identifying only one trigger (53\%) and thirty-one patients with multiple identified triggers (29\%). Twenty patients had unknown triggers (19\%). Interestingly, only four patients in the study identified the intake of ULTs allopurinol and febuxostat as a trigger for an acute flare (4\%). The most commonly identified flare triggers were food (63\%), alcohol beverages (29\%), and hospitalization (9\%). This was consistent with previous data that diet and alcohol consumption, especially that of meat intake, sugarsweetened soft drinks, high-fructose foods, beer, and hard liquor, increase the risk of incident gout. ${ }^{17,18} \mathrm{~A}$ New Zealand study by Flynn et al. in 2015 had similar results. Men and women with gout were surveyed, and $70.6 \%$ self-reported $\geq 1$ food or drink trigger acute gout attacks. The top 3 reported triggers were seafood or fish (62.5\%), alcohol (47.1\%), and red meat (35.2\%). ${ }^{33}$ This is in contrast to a 2017 study in the United Kingdom by Abhishek et al., where more than $60 \%$ of patients had no identifiable trigger. $26 \%$ of patients identified only one trigger, and $11.6 \%$ of patients reported multiple triggers. The most frequently self-reported triggers were alcohol beverages (14.2\%), dehydration (4.9\%), injury or excess activity (4.9\%), excessively warm or cold weather (4.4\% and 5.5\% respectively), and red-meat (4.4\%). ${ }^{12}$ The variability of flare triggers among the different studies may be due to genetic, environmental, and, lifestyle and diet differences between the populations. As previously put forth by Abhishek, these studies may imply that flare triggers are person-specific. People with gout should be advised to avoid the factors they recognize may trigger their gout attacks rather than be concerned with all potential triggers.

All risk factors for frequent attacks, namely onset of symptoms before age 40, male sex, hypertension, high BMI, and in particular, elevated uric acid levels, were not significantly different across clinical-stage scenarios in our cohort. This contrasts with the findings of Rashid et al., where SUA level was lowest in the 0 gout flare group $(8.73 \pm 1.5$ $\mathrm{mg} / \mathrm{dL})$ and highest in the $\geq 3$ gout flare group $(9.26 \pm 1.8$ $\mathrm{mg} / \mathrm{dL}) .^{32}$ Their findings suggest that younger patients were more likely to have $1-2$ gout flares, and patients $\geq 65$ years of age had $\geq 3$ gout flares. Our study's sample size may be too small to conclude which risk factors are seen in a particular clinical-stage scenario.

\section{Management}

Most patients were managed on an outpatient basis with colchicine, followed by allopurinol and febuxostat. Of note, only $87 \%$ of patients were prescribed colchicine, NSAIDs, or oral steroids. Possibly, the frequent presence of comorbidities such as chronic kidney disease, ischemic heart disease, hypertension, and diabetes limits the use of these anti-inflammatory drugs. Interestingly, not all patients were prescribed ULTs after consultation. Only $75 \%$ of patients were prescribed ULT with allopurinol ordered more often than febuxostat (45\% vs. $30 \%)$. Several factors may explain this: recency or frequency of an attack or both, presence of chronic kidney disease, or the cost of medications. Poor follow-up compliance and sub-optimal use of ULT were 
also observed in other studies. They found that patients do not perceive allopurinol to improve their quality of life. Many are unaware of the rationale behind ULT, with many discontinuing treatments at the onset of flares when ULT is initiated. ${ }^{21}$ In our study, a possible explanation for such poor compliance could be the finding that only $16 \%$ of the cohort were given patient education.

\section{CONCLUSION}

In conclusion, approximately $70 \%$ of the study patients already had tophi classifying them as CCS $4-9$, while $30 \%$ were CCS 1-3. Being a tertiary care center, this may explain the longstanding and more severe disease associated with CCS 4-9 seen in the study.

The demographic profile is similar to published data. The majority are males with the onset of symptoms in their 40's. Comorbidities are identical to world figures but differ in the low incidence of obesity and metabolic syndrome. Comparing the Filipino clinical profile to historical data suggests an increased incidence of gout in the young and an increase in comorbidity prevalence. This cohort also differs from literature in having the ankle as the most common initial joint presentation. Treatment given to the majority reflects standard gout management and what is available in the Philippines. In this study population, we note that most have normal BMI and are smokers, which is not seen in other gout patient populations.

While analysis of CCS progression as a function of time shows no significant difference, the trend of low to high CCS due to delays in early consultation, referral and diagnosis highlight the need for patient education and a high index of suspicion for physicians and specialists.

This pilot study's intention, which the authors have identified as a gout registry feasibility study, is recommended to be adapted to different settings. The difficulties, nuances experienced, and expenses incurred were noted during the study period. Method of questioning, data collection, and questionnaire changes were made and implemented in continuing research.

Creating a dedicated gout registry will provide physicians, caregivers, and policymakers information on the prevention, diagnosis, management of gout, and where there is a need for future studies. It will also form the foundation for modernizing the Philippine CPG and its upkeep. In the long term, it will improve the quality of care of patients and prevent complications. As a pilot study for a registry, this study can be implemented in different Philippine settings to broaden and monitor the ever-changing Filipino gout profile.

\section{Recommendation}

A larger sample size and a more extended observation period are recommended to estimate the prevalence of the CCS of gout, risk factors for flares, and response to treatment.
Other outcomes that can be measured with a more extended study period are mortality rates and etiologies per CCS. Questionnaire revision to streamline data collection will make patient interviews and physical examinations more efficient. A manual of procedures may be developed to standardize and reduce errors once the gout registry is open to other institutions.

\section{Statement of Authorship}

All authors participated in the data collection and analysis and approved the final version submitted.

\section{Author Disclosure}

All authors declared no conflicts of interest.

\section{Funding Source}

This paper was self-funded.

\section{REFERENCES}

1. Khanna D, Fitzgerald JD, Khanna PP, Bae S, Singh MK, Neogi T, et al. American College of Rheumatology. 2012 American College of Rheumatology guidelines for management of gout. Part 1: systematic nonpharmacologic and pharmacologic therapeutic approaches to hyperuricemia. Arthritis Care Res (Hoboken). 2012 Oct;64(10):1431-46. doi: 10.1002/acr.21772. PMID: 23024028; PMCID: PMC3683400.

2. Neogi T, Jansen TL, Dalbeth N, Fransen J, Schumacher HR, Berendsen D, et al. 2015 Gout classification criteria: an American College of Rheumatology/European League Against Rheumatism collaborative initiative. Ann Rheum Dis. 2015 Oct;74(10):1789-98. doi: 10.1136/annrheumdis-2015-208237. Erratum in: Ann Rheum Dis. 2016 Feb;75(2):473. PMID: 26359487; PMCID: PMC4602275.

3. Dans LS, Salido EO, Penserga EG NS. National Nutrition and Health Survey: Prevalence of rheumatic diseases among adult Filipinos. Phil J Int Medicine. 2006;(44):297-303.

4. Prasad P, Krishnan E. Filipino gout: a review. Arthritis Care Res (Hoboken). 2014 Mar;66(3):337-43. doi: 10.1002/acr.22118. PMID: 23983155.

5. Singh JA, Reddy SG, Kundukulam J. Risk factors for gout and prevention: a systematic review of the literature. Curr Opin Rheumatol. 2011 Mar;23(2):192-202. doi: 10.1097/BOR.0b013e3283438e13. PMID: 21285714; PMCID: PMC4104583.

6. Ragab G, Elshahaly M, Bardin T. Gout: An old disease in new perspective - A review. J Adv Res. 2017 Sep;8(5):495-511. doi: 10.1016/j.jare.2017.04.008. Epub 2017 May 10. PMID: 28748116; PMCID: PMC5512152.

7. Mumford SL, Dasharathy SS, Pollack AZ, Perkins NJ, Mattison DR, Cole SR, et al. Serum uric acid in relation to endogenous reproductive hormones during the menstrual cycle: findings from the BioCycle study. Hum Reprod. 2013 Jul;28(7):1853-62. doi: 10.1093/humrep/ det085. Epub 2013 Apr 5. PMID: 23562957; PMCID: PMC3685334.

8. Takiue $Y$, Hosoyamada M, Kimura M, Saito $H$. The effect of female hormones upon urate transport systems in the mouse kidney. Nucleosides Nucleotides Nucleic Acids. 2011 Feb;30(2):113-9. doi: 10.1080/15257770.2010.551645. PMID: 21360409.

9. Kuo CF, Grainge MJ, Zhang W, Doherty M. Global epidemiology of gout: prevalence, incidence and risk factors. Nat Rev Rheumatol. 2015 Nov;11(11):649-62. doi: 10.1038/nrrheum.2015.91. Epub 2015 Jul 7. PMID: 26150127.

10. Kim JW, Kwak SG, Lee H, Kim SK, Choe JY, Park SH. Prevalence and incidence of gout in Korea: data from the national health claims database 2007-2015. Rheumatol Int. 2017 Sep;37(9):1499-1506. doi: 10.1007/s00296-017-3768-4. Epub 2017 Jul 4. PMID: 28676911. 
11. Chen SY, Shen ML. Juvenile gout in Taiwan associated with family history and overweight. J Rheumatol. 2007 Nov;34(11):2308-11. Epub 2007 Oct 15. PMID: 17937457.

12. Abhishek A, Valdes AM, Jenkins W, Zhang W, Doherty M. Triggers of acute attacks of gout, does age of gout onset matter? A primary care based cross-sectional study. PLoS One. 2017 Oct 12;12(10):e0186096. doi: 10.1371/journal.pone.0186096. PMID: 29023487; PMCID: PMC5638318.

13. Kuo CF, Grainge MJ, Mallen C, Zhang W, Doherty M. Comorbidities in patients with gout prior to and following diagnosis: case-control study. Ann Rheum Dis. 2016 Jan;75(1):210-7. doi: 10.1136/ annrheumdis-2014-206410. Epub 2014 Nov 14. PMID: 25398375; PMCID: PMC4717388.

14. Li-Yu J, Salido E, Manahan S, Lichauco J, Lorenzo J. Philippine Clinical Practice Guidelines for the Management of Gout. Philippine Journal of Internal Medicine. 2008;46.

15. Khanna D, Khanna PP, Fitzgerald JD, Singh MK, Bae S, Neogi T, et al. American College of Rheumatology. 2012 American College of Rheumatology guidelines for management of gout. Part 2: therapy and antiinflammatory prophylaxis of acute gouty arthritis. Arthritis Care Res (Hoboken). 2012 Oct;64(10):1447-61. doi: 10.1002/acr.21773. PMID: 23024029; PMCID: PMC3662546.

16. Roughley MJ, Belcher J, Mallen CD, Roddy E. Gout and risk of chronic kidney disease and nephrolithiasis: meta-analysis of observational studies. Arthritis Res Ther. 2015 Apr 1;17(1):90. doi: 10.1186/s13075015-0610-9. PMID: 25889144; PMCID: PMC4404569.

17. Singh JA, Ramachandaran R, Yu S, Yang S, Xie F, Yun H, et al. Is gout a risk equivalent to diabetes for stroke and myocardial infarction? A retrospective claims database study. Arthritis Res Ther. 2017 Oct 17;19(1):228. doi: 10.1186/s13075-017-1427-5. PMID: 29041963; PMCID: PMC5646136.

18. Singh JA, Bharat A, Khanna D, Aquino-Beaton C, Persselin JE, Duffy E, et al. Racial differences in health-related quality of life and functional ability in patients with gout. Rheumatology (Oxford). 2017 Jan;56(1):103-112. doi: 10.1093/rheumatology/kew356. Epub 2016 Oct 25. PMID: 28028159; PMCID: PMC5188996.

19. American Board of Medical Specialties, NQRN National Quality Registry Network: What is a Clinical Registry [Internet] available from: https://www.abms.org/media/1358/what-is-a-clinical-dataregistry.pdf

20. Taylor WJ, Fransen J, Dalbeth N, et al. Performance of classification criteria for gout in early and established disease. Ann Rheum Dis. 2016;75(1):178-182. doi:10.1136/annrheumdis-2014-206364

21. Chandratre P, Roddy E, Clarson L, Richardson J, Hider SL, Mallen CD. Health-related quality of life in gout: a systematic review. Rheumatology (Oxford). 2013 Nov;52(11):2031-40. doi: 10.1093/ rheumatology/ket265. Epub 2013 Aug 11. PMID: 23934311; PMCID: PMC3798715.

22. Roddy E, Zhang W, Doherty M. The changing epidemiology of gout. Nat Clin Pract Rheumatol. 2007 Aug;3(8):443-9. doi: 10.1038/ ncprheum0556. PMID: 17664951.
23. Pisaniello HL, Lester S, Gonzalez-Chica D, Stocks N, Longo M, Sharplin GR, et al. Gout prevalence and predictors of urate-lowering therapy use: results from a population-based study. Arthritis Res Ther. 2018 Jul 11;20(1):143. doi: 10.1186/s13075-018-1633-9. PMID: 29996922; PMCID: PMC6042461.

24. MacFarlane LA, Kim SC. Gout: a review of nonmodifiable and modifiable risk factors. Rheum Dis Clin North Am. 2014 Nov;40(4):581-604. doi: 10.1016/j.rdc.2014.07.002. Epub 2014 Sep 2. PMID: 25437279; PMCID: PMC4251556.

25. Maynard JW, McAdams DeMarco MA, Baer AN, Köttgen A, Folsom AR, Coresh J, Gelber AC. Incident gout in women and association with obesity in the Atherosclerosis Risk in Communities (ARIC) Study. Am J Med. 2012 Jul;125(7):717.e9-717.e17. doi: 10.1016/j. amjmed.2011.11.018. Epub 2012 May 8. PMID: 22571781; PMCID: PMC3383456.

26. McAdams-DeMarco MA, Maynard JW, Baer AN, Coresh J. Hypertension and the risk of incident gout in a populationbased study: the atherosclerosis risk in communities cohort. J Clin Hypertens (Greenwich). 2012 Oct;14(10):675-9. doi: 10.1111/j.17517176.2012.00674.x. Epub 2012 Jun 13. PMID: 23031144; PMCID: PMC3464949.

27. Krishnan E. Chronic kidney disease and the risk of incident gout among middle-aged men: a seven-year prospective observational study. Arthritis Rheum. 2013 Dec;65(12):3271-8. doi: 10.1002/art.38171. PMID: 23982888.

28. Abeles AM. Hyperuricemia, gout, and cardiovascular disease: an update. Curr Rheumatol Rep. 2015 Mar;17(3):13. doi: 10.1007/ s11926-015-0495-2. PMID: 25740704

29. Seminog OO, Goldacre MJ. Gout as a risk factor for myocardial infarction and stroke in England: evidence from record linkage studies. Rheumatology (Oxford). 2013 Dec;52(12):2251-9. doi: 10.1093/ rheumatology/ket293. Epub 2013 Sep 17. PMID: 24046469.

30. Liu SC, Xia L, Zhang J, Lu XH, Hu DK, Zhang HT, Li HJ. Gout and Risk of Myocardial Infarction: A Systematic Review and MetaAnalysis of Cohort Studies. PLoS One. 2015 Jul 31;10(7):e0134088. doi: 10.1371/journal.pone.0134088. PMID: 26230580; PMCID: PMC4521845.

31. Torralba TP, Bayani-Sioson PS. The Filipino and gout. Semin Arthritis Rheum. 1975;4:307-20.

32. Rashid N, Levy GD, Wu YL, Zheng C, Koblick R, Cheetham TC. Patient and clinical characteristics associated with gout flares in an integrated healthcare system. Rheumatol Int. 2015 Nov;35(11): 1799-807. doi: 10.1007/s00296-015-3284-3. Epub 2015 May 20. PMID: 25991397; PMCID: PMC4611012.

33. Flynn TJ, Cadzow M, Dalbeth N, Jones PB, Stamp LK, Hindmarsh $\mathrm{JH}$, et al. Positive association of tomato consumption with serum urate: support for tomato consumption as an anecdotal trigger of gout flares. BMC Musculoskelet Disord. 2015 Aug 19;16:196. doi: 10.1186/s12891-015-0661-8. PMID: 26286027; PMCID: PMC4541734. 


\section{APPENDICES}

\section{Appendix A. 2015 ACR-EULAR Gout Classification Criteria}

Table 2 The ACR/EULAR gout classification criteria*

\begin{tabular}{ll}
\hline Step 1: Entry criterion (only apply criteria below to those meeting this entry criterion) & $\begin{array}{l}\text { Categories } \\
\text { At least 1 episode of swelling, pain, or tenderness in a peripheral joint } \\
\text { or bursa }\end{array}$ \\
Step 2: Sufficient criterion (if met, can classify as gout without applying criteria below) & $\begin{array}{l}\text { Presence of MSU crystals in a symptomatic joint or bursa (ie, in } \\
\text { synovial fluid) or tophus }\end{array}$ \\
Step 3: Criteria (to be used if sufficient criterion not met) &
\end{tabular}

Step 3: Criteria (to be used if sufficient criterion not met)

Clinical

Pattern of joint/bursa involvement during symptomatic episode(s) ever

Ankle or mid-foot (as part of monoarticular or oligoarticular episode without involvement of the first metatarsophalangeal joint

Involvement of the first metatarsophalangeal joint (as part of monoarticular or oligoarticular episode)

Characteristics of symptomatic episode(s) ever

- Erythema overlying affected joint (patient-reported or physician-observed)

- Can't bear touch or pressure to affected joint

- Great difficulty with walking or inability to use affected joint

One characteristic

Two characteristics

Three characteristics

Time course of episode(s) ever

Presence (ever) of $\geq 2$, irrespective of anti-inflammatory treatment:

- Time to maximal pain $<24 \mathrm{~h}$

- Resolution of symptoms in $\leq 14$ days

- Complete resolution (to baseline level) between symptomatic episodes

One typical episode

Recurrent typical episodes

Clinical evidence of tophus

Draining or chalk-like subcutaneous nodule under transparent skin, often with overlying vascularity, located in typical locations: joints, ears, olecranon bursae, finger pads, tendons (eg, Achilles)

Laboratory

Serum urate: Measured by the uricase method.

Ideally should be scored at a time when the patient was not receiving urate-lowering treatment and it was $>4$ weeks from the start of an episode (ie, during the intercritical period); if practicable, retest under those conditions. The highest value irrespective of timing should be scored

Synovial fluid analysis of a symptomatic (ever) joint or bursa (should be assessed by a trained observer) $\ddagger$

Present

MSU negative

Imaging $\S$

Imaging evidence of urate deposition in symptomatic (ever) joint or bursa: ultrasound evidence of double-contour sign or DECT demonstrating urate deposition**

Present (either modality)

Imaging evidence of gout-related joint damage: conventional radiography of the hands and/or feet demonstrates at least 1 erosiont $\dagger$

Present

${ }^{*}$ A web-based calculator can be accessed at: http://goutclassificationcalculator.auckland.ac.nz, and through the American College of Rheumatology (ACR) and European League Against Rheumatism (EULAR) web sites.

tSymptomatic episodes are periods of symptoms that include any swelling, pain, and/or tenderness in a peripheral joint or bursa.

flf serum urate level is $<4 \mathrm{mg} / \mathrm{dL}(<0.24 \mathrm{mmoles} /$ liter), subtract 4 points; if serum urate level is $>4 \mathrm{mg} / \mathrm{dL}->6 \mathrm{mg} / \mathrm{dL}$ ( $>0.24-<0.36 \mathrm{mmoles} /$ liter), score this item as 0 .

§If polarizing microscopy of synovial fluid from a symptomatic (ever) joint or bursa by a trained examiner fails to show monosodium urate monohydrate (MSU) crystals, subtract 2 points.

If synovial fluid was not assessed, score this item as 0.

qlf imaging is not available, score these items as 0 .

\#Hyperechoic irregular enhancement over the surface of the hyaline cartilage that is independent of the insonation angle of the ultrasound beam (note: false-positive double-contour sign [artifact] may appear at the cartilage surface but should disappear with a change in the insonation angle of the probe). ${ }^{31} 32$

**Presence of color-coded urate at articular or periarticular sites. Images should be acquired using a dual-energy computed tomography (DECT) scanner, with data acquired at $80 \mathrm{kV}$ and $140 \mathrm{kV}$ and analyzed using gout-specific software with a 2-material decomposition algorithm that color-codes urate. ${ }^{33}$ A positive scan is defined as the presence of color-coded urate at articular or periarticular sites. Nailbed, submillimeter, skin, motion, beam hardening, and vascular artifacts should not be interpreted as DECT evidence of urate deposition. ${ }^{34}$

t+Erosion is defined as a cortical break with sclerotic margin and overhanging edge, excluding distal interphalangeal joints and gull wing appearance.

Neogi T, Jansen TLTA, Dalbeth N, et al. 2015 Gout classification criteria: An American College of Rheumatology/European League Against Rheumatism collaborative initiative. Annals of the Rheumatic Diseases. 2015;74(10):1789-1798. doi:10.1136/annrheumdis-2015-208237 


\section{Appendix B. 2012 ACR Gout Clinical Case Scenarios}

Table 1. Gout Case Scenarios

\begin{tabular}{|c|c|c|c|}
\hline Symptoms & $\begin{array}{l}\text { Tophus or Tophi } \\
\text { detected on } \\
\text { Physical Exam }\end{array}$ & Frequency & $\begin{array}{c}\text { CASE } \\
\text { SCENARIO } \\
\text { NUMBER }\end{array}$ \\
\hline \multirow{3}{*}{$\begin{array}{l}\text { Intermittent } \\
\text { symptoms }\end{array}$} & NO & Infrequent Symptoms ( $\leq 1$ attack/yr) & 1 \\
\hline & NO & Frequent Symptoms (2-6 attacks/yr) & 2 \\
\hline & NO & Very Frequent Symptoms (> 7 attacks/yr) & 3 \\
\hline \multirow[t]{3}{*}{$\begin{array}{l}\text { Intermittent } \\
\text { symptoms }\end{array}$} & YES & Infrequent Symptoms ( $\leq 1$ attack/yr) & 4 \\
\hline & YES & Frequent Symptoms (2-6 attacks/yr) & 5 \\
\hline & YES & Very Frequent Symptoms (> 7 attacks/yr) & 6 \\
\hline
\end{tabular}

Table 2. Case Scenarios for Chronic Tophaceous Gouty Arthropathy (CTGA)

\begin{tabular}{|c|l|c|}
\hline $\begin{array}{c}\text { Disease } \\
\text { Severity }\end{array}$ & \multicolumn{1}{|c|}{ Characteristics } & $\begin{array}{c}\text { CASE } \\
\text { SCENARIO } \\
\text { NUMBER }\end{array}$ \\
\hline Mild & $\begin{array}{l}\cdot \text { Simple chronic tophaceous gouty arthropathy } \\
- \text { Affecting 1 joint } \\
\cdot \text { Stable disease }\end{array}$ & 7 \\
\hline Moderate & $\begin{array}{l}\cdot \text { Simple chronic tophaceous gouty arthropathy } \\
- \text { Affecting 2-4 joints }\end{array}$ & 8 \\
\hline Severe & $\begin{array}{l}\cdot \text { Stable disease } \\
\text { OR } \\
-\geq 1 \text { unstable, complicated, severe articular tophus or tophi }\end{array}$ & 9 \\
\hline
\end{tabular}

Fundamental case scenarios evaluated by the task force panel (TFP). The TFP evaluated a broad spectrum of severity of gout, with presenting clinical information comparable to that encountered in practice. Scenarios were formulated iteratively by the core expert panel, as described in the text, and were not intended to serve as disease classification criteria. All case scenarios assumed that the diagnosis of gout was correct, and that there was some evidence of gout disease activity. Three distinct "treatment groups" for these recommendations, each with 3 case scenarios designed to succinctly represent clinically-based decision making and totaling 9 in all, are shown. The treatment group with intermittent attacks of acute gout but no tophi detected on physical examination was subdivided based on increasing yearly frequency of episodes of acute gouty arthritis of at least moderate to severe pain intensity (case scenarios 1-3; A). Gout associated with clinically apparent high body urate burden was evaluated in case scenarios where there were _1 tophi on physical examination, and either A, intermittently symptomatic acute gouty arthritis (case scenarios 4-6), or B, chronic joint symptoms due to synovitis attributable to gout or articular tophus or tophi in case scenarios 7-9 (the domain termed chronic tophaceous gouty arthropathy [CTGA]). Severity of case scenarios in the CTGA domain was distinguished by extent and characteristics of the tophi and chronic arthropathy, with variable inflammatory and deforming features detected on physical examination.

Khanna D, Fitzgerald JD, Khanna PP, et al. 2012 American College of Rheumatology guidelines for management of gout. Part 1: systematic nonpharmacologic and pharmacologic therapeutic approaches to hyperuricemia. Arthritis Care Res (Hoboken). 2012;64(10):1431-1446. 